Ser ot oni $n$ neur ons in the dor sal $r$ aphe medi at $e$ the ant $i c a t a p l e c t i c$ act $i$ on of oxi $n$ neurons by reducing anygdal a activity

\begin{tabular}{|l|l|}
\hline 著者 & $\begin{array}{l}\text { Hasegawa Emi, Naej i ma Takashi, Yoshi da } \\
\text { Takayuki, Nasseck O i vi a A., Her I i t ze St ef an, } \\
\text { Yoshi oka M t suhi ro, Sakur ai Takeshi, M eda } \\
\text { M chi hi ro }\end{array}$ \\
\hline $\begin{array}{l}\text { j our nal or } \\
\text { publ i cat i on t i tl e }\end{array}$ & $\begin{array}{l}\text { Proceedi ngs of the Nat i onal Acadeny of } \\
\text { Sci ences of the Uni ted St at es of Amer i ca }\end{array}$ \\
\hline vol une & 114 \\
\hline nunber & 17 \\
\hline page r ange & E3526- E3535 \\
\hline year & $2017-$ O4 25 \\
\hline URL & ht t p: //hdl . handl e. net /2297/47896 \\
\hline
\end{tabular}




\title{
Serotonin neurons in the dorsal raphe mediate the anticataplectic action of orexin neurons by reducing amygdala activity
}

\author{
Emi Hasegawa ${ }^{a, 1}$, Takashi Maejimaa $^{a}$, Takayuki Yoshidab ${ }^{b}$ Olivia A. Masseckc, Stefan Herlitzec, Mitsuhiro Yoshioka ${ }^{b}$, \\ Takeshi Sakurai ${ }^{\mathrm{a}, 1}$, and Michihiro Mieda ${ }^{\mathrm{a}, 2}$ \\ aDepartment of Molecular Neuroscience and Integrative Physiology, Graduate School of Medical Sciences, Kanazawa University, Kanazawa 920-8640,
Japan; ${ }^{b}$ Department of Neuropharmacology, Hokkaido University Graduate School of Medicine, Sapporo 060-8638, Japan; and 'Department of General
Zoology and Neurobiology, Ruhr-University Bochum, 44780 Bochum, Germany
}

Edited by Joseph S. Takahashi, Howard Hughes Medical Institute, University of Texas Southwestern Medical Center, Dallas, TX, and approved March 20, 2017 (received for review August 31, 2016)

\begin{abstract}
Narcolepsy is a sleep disorder caused by the loss of orexin (hypocretin)producing neurons and marked by excessive daytime sleepiness and a sudden weakening of muscle tone, or cataplexy, often triggered by strong emotions. In a mouse model for narcolepsy, we previously demonstrated that serotonin neurons of the dorsal raphe nucleus (DRN) mediate the suppression of cataplexy-like episodes (CLEs) by orexin neurons. Using an optogenetic tool, in this paper we show that the acute activation of DRN serotonin neuron terminals in the amygdala, but not in nuclei involved in regulating rapid eye-movement sleep and atonia, suppressed CLEs. Not only did stimulating serotonin nerve terminals reduce amygdala activity, but the chemogenetic inhibition of the amygdala using designer receptors exclusively activated by designer drugs also drastically decreased CLEs, whereas chemogenetic activation increased them. Moreover, the optogenetic inhibition of serotonin nerve terminals in the amygdala blocked the anticataplectic effects of orexin signaling in DRN serotonin neurons. Taken together, the results suggest that DRN serotonin neurons, as a downstream target of orexin neurons, inhibit cataplexy by reducing the activity of amygdala as a center for emotional processing.
\end{abstract}

sleep | narcolepsy | orexin | serotonin | amygdala

$\mathbf{N}$ arcolepsy type 1 (narcolepsy with cataplexy) is a debilitating sleep disorder caused by degenerative loss of neurons producing the neuropeptides orexin A and orexin B (orexin neurons) (1-3). Its symptoms can be divided into two independent pathological phenomena $(4,5)$. The first phenomenon is dysregulation of nonrapid eye-movement (NREM) sleep onset: the inability to maintain a consolidated awake period that is characterized by abrupt transitions from wakefulness to NREM sleep. This phenomenon manifests itself clinically as excessive daytime sleepiness or sleep attacks. The second phenomenon is dysregulation of rapid eye-movement (REM) sleep onset: the pathological intrusion of REM sleep or REM atonia at sleep onset or into wakefulness. It is during these periods that patients may experience hypnagogic hallucinations, sleep paralysis, and cataplexy. Cataplexy manifests as sudden episodes of muscle weakness without consciousness impairment triggered by strong emotions of positive valence, including laughter, joking, and delight.

Similarly, mice lacking orexin peptides, orexin neurons, or orexin receptors recapitulate human narcolepsy type 1 phenotypes to further highlight the critical role that orexin signaling plays in the maintenance of wakefulness (5-7). The mice demonstrate markedly decreased duration of wakefulness episodes during the dark phase (i.e., inability to maintain a long awake period, or sleepiness), abrupt behavioral arrests with muscle atonia that manifest as direct transitions from wakefulness to REM sleep in electroencephalogram/ electromyogram (EEG/EMG) recordings (i.e., cataplexy), decreased REM sleep latency, and increased REM sleep time during the dark phase.
Orexin neurons are distributed within the lateral hypothalamus and send projections throughout the brain and spinal cord. There are particularly dense innervations to nuclei containing monoaminergic and cholinergic neurons that constitute the ascending activating system in the brainstem and the hypothalamus $(5,6)$. We previously elucidated two critical efferent pathways of orexin neurons; serotonin neurons in the dorsal raphe nucleus (DRN) inhibit cataplexy-like episodes (CLEs), whereas noradrenergic neurons in the locus coeruleus (LC) stabilize wakefulness episodes (8). In that study, we called direct transitions from wakefulness to REM sleep shown in EEG/EMG data CLEs, in reference to Scammell et al. (9), which were nearly equivalent to murine cataplexy, although the mice were not videotaped to confirm their immobility during such episodes.

To further clarify the pathophysiology of narcolepsy and regulation of sleep/wakefulness by orexin neurons, we aimed to identify the downstream target that mediates the anticataplectic effects of serotonin neurons in the DRN that also send widespread projections throughout the brain (10-12). Based on evidence using electrophysiological, neurochemical, genetic, and neuropharmacological approaches, DRN serotonin neurons have long been implicated in promoting wakefulness and suppressing REM sleep $(13,14)$. Along with regulating sleep/wakefulness, serotonin in the brain has been

\section{Significance}

Although the neurodegeneration of orexin (hypocretin)-producing neurons clearly causes the sleep disorder narcolepsy, the precise neural mechanisms by which orexin neurons prevent narcolepsy remain unclear. We previously demonstrated that orexin neurons inhibit cataplexy-like episodes-cataplexy is a cardinal symptom of narcolepsy, characterized by a sudden weakening of muscle tonevia serotonin neurons in the dorsal raphe nucleus (DRN). We thus used optogenetic and chemogenetic approaches to demonstrate that DRN serotonin neurons suppress cataplexy-like episodes by reducing the activity of the amygdala that plays an important role in emotional processing, as consistent with the fact that strong emotions often trigger cataplexy. We therefore propose that the orexin neuron-DRN serotonin neuron-amygdala pathway is a critical circuit for preventing cataplexy.

Author contributions: E.H., T.M., and M.M. designed research; E.H., T.M., T.Y., M.Y., and M.M. performed research; T.M., O.A.M., S.H., and T.S. contributed new reagents/analytic tools; E.H., T.M., T.Y., M.Y., and M.M. analyzed data; and E.H., T.M., and M.M. wrote the paper.

The authors declare no conflict of interest.

This article is a PNAS Direct Submission.

${ }^{1}$ Present address: International Institute for Integrative Sleep Medicine, University of Tsukuba, Tsukuba 305-8575, Japan.

${ }^{2}$ To whom correspondence should be addressed. Email: mieda@med.kanazawa-u.ac.jp.

This article contains supporting information online at www.pnas.org/lookup/suppl/doi:10. 1073/pnas.1614552114/-/DCSupplemental. 
implicated in various sensorimotor, affective, and cognitive behaviors (15-18). Therefore, identifying the action site of DRN serotonin neurons in preventing CLEs can shed additional light on the multiple functions of the serotonin system.

\section{Results}

Optogenetic Activation of Serotonin Neurons in the DRN Suppresses CLEs in Narcoleptic orexin-ataxin3 Mice. We previously demonstrated that serotonin neurons of the DRN mediate an anticataplectic action of orexin neurons (8). To further confirm the physiological importance of orexinergic activation in DRN serotonin neurons, we compared the activity of DRN serotonin neurons between control and orexin neuron-deficient (orexinataxin3) mice (7) by measuring Fos protein expression. To increase cataplexy, we gave chocolate to the mice beginning at the onset of the dark phase (19), as shown in Fig. S1. Fos expression in the tryptophan hydroxylase 2 (TPH2)-positive serotonin neurons of the DRN after 90 min of chocolate feeding was significantly lower in orexin-ataxin 3 mice than in control mice (Fig. 1). That result suggests that orexin neurons indeed enhance the

\section{ADRN : Control}
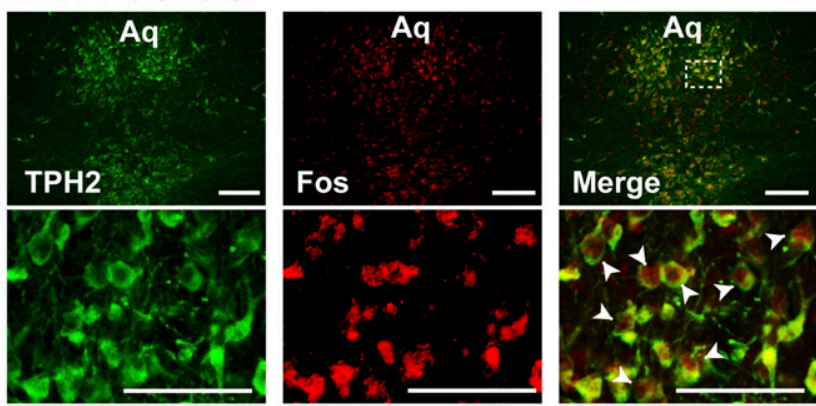

\section{DRN : Orexin-Ataxin3}
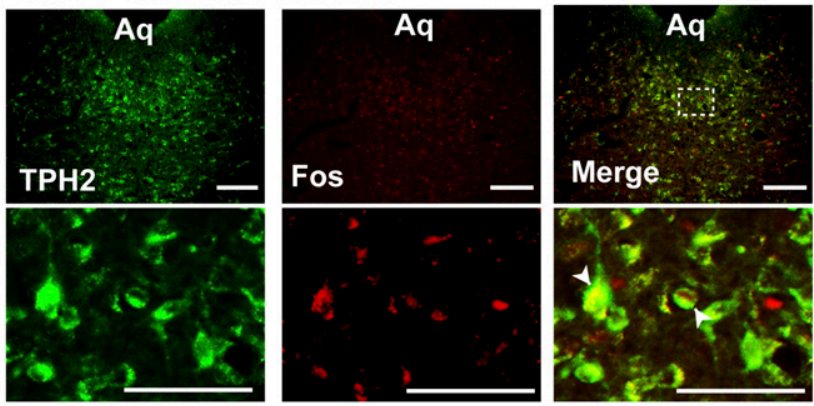

B
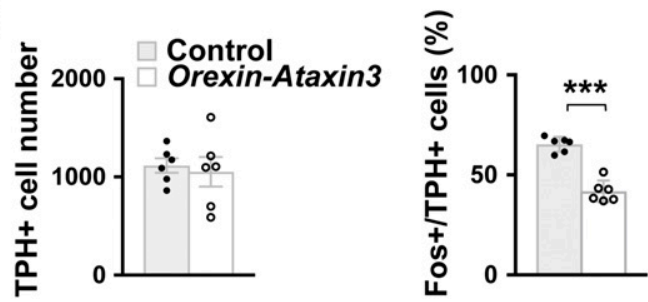

Fig. 1. Activity of DRN serotonin neurons is reduced in orexin neuron-deficient orexin-ataxin3 mice. (A) Coronal brain sections containing the DRN prepared from Sert-Cre (Control) or Sert-Cre;orexin-ataxin3 (Orexin-Ataxin3) mice after 90 min of chocolate feeding at the onset of the dark phase were double-stained with antiTPH (green) and anti-Fos (red) antibodies. Regions outlined with dashed lines are shown at higher magnification. Fos ${ }^{+} \mathrm{TPH}^{+}$cells are indicated by white arrowheads. Aq, aqueduct. (Scale bars, $100 \mu \mathrm{m}$.) $(B)$ The number of Fos ${ }^{+} \mathrm{TPH}^{+}$cells (expressed as a percentage of total $\mathrm{TPH}^{+}$cells) decreased, whereas the total number of TPH $2^{+}$cells did not change in Sert-Cre;orexin-ataxin3 mice $(n=6)$. Bar graphs show mean \pm SEM, $* * \star P<0.001$, two-tailed Student's $t$ test. activity of serotonin neurons directly or indirectly, if not both, as consistent with the role of orexin signaling in DRN serotonin neurons in inhibiting CLEs.

Knowing that DRN serotonin neurons send projections throughout the brain (10-12), we aimed to determine the site of the anticataplectic action of those neurons: that is, the downstream targets of DRN serotonin neurons in suppressing CLEs. To that end, we used channelrhodopsin-2 (ChR2) to activate the neurons because axonal projections of ChR2-expressing neurons can be selectively activated at each target site (20). To minimize light illumination, we used a variant of ChR2-namely, the stabilized step-function opsin (SSFO) (21) - whose activation with a single blue-light pulse increases the excitability of targeted cells during depolarization sustained for more than $30 \mathrm{~min}$. Accordingly, we expressed SSFO in serotonin neurons of the DRN by stereotaxically microinjecting a Cre-inducible adeno-associated virus (AAV) vector (20) AAV-EF1 $\alpha$-DIO-SSFO-EYFP (DIO: double-floxed inverted open-reading-frame) in the DRN of orexin-ataxin 3 mice crossed with Sert-Cre mice (22), which expresses Cre recombinase specifically in serotonin neurons (SertCre;orexin-ataxin3 mice). In those mice, SSFO-EYFP was expressed in $92.3 \pm 3.62 \%$ of $\mathrm{TPH}_{2}{ }^{+}$cells, and $100 \%$ of $\mathrm{EYFP}^{+}$ cells were $\mathrm{TPH}_{2}{ }^{+}(n=5)$, which confirms the targeted expression of SSFO in serotonin neurons (Fig. $2 A$ ).

Next, we analyzed the sleep/wakefulness patterns of the mice. To increase the frequency of CLE, we gave mice chocolate for the first $4 \mathrm{~h}$ of the dark phase (19). We first inserted an optic fiber above the DRN to stimulate the cell bodies of serotonin neurons. Without optogenetic stimulation, Sert-Cre;orexin-ataxin3 mice expressing SSFO in DRN serotonin neurons exhibited several CLEs during chocolate feeding (Fig. $2 B$ ). The EEG of CLEs was dominated by $\theta$-activity and closely resembled that of cataplexy-associated states previously reported (23), which were enriched during murine cataplexy and distinguished from REM sleep by a larger, highly irregular amplitude (Fig. S2 $A$ and $B$ ). When DRN serotonin neurons were optogenetically stimulated during the chocolate-feeding period by applying blue light ( $473 \mathrm{~nm}, 10-\mathrm{ms}$ pulses, $5 \mathrm{~Hz}, 1 \mathrm{~s})$ every 30 min (Fig. S1A), CLEs were drastically suppressed, whereas the fragmentation of wakefulness episodes (i.e., reduced duration of wakefulness episodes) remained unaffected (Fig. $2 B$ and $D$ ). Time spent in wakefulness, NREM sleep, and REM sleep did not change considerably (Fig. $2 C$ and Table S1). We also confirmed that the activation of SSFO indeed elevated the activity of those neurons ex vivo via loose patch-clamp recordings on DRN slices and in vivo via measurements of the expression of the Fos protein (Fig. $2 E-G$ ). We thus optogenetically reproduced the anticataplectic activity of DRN serotonin neurons that we had previously demonstrated via a chemogenetic approach using hM3Dq $(8,24)$, an excitatory designer receptor exclusively activated by designer drugs (DREADD).

Optogenetic Activation of DRN Serotonin Projections in the Amygdala Suppresses CLEs in orexin-ataxin3 Mice. We optogenetically activated SSFO-expressing DRN serotonin terminals to identify the downstream targets of those neurons in suppressing CLEs. When brain sections of Sert-Cre mice with an AAV-EF1 $\alpha$-DIO-SSFOEYFP injection in the DRN were immunostained with an antiGFP antibody, we observed $\mathrm{EYFP}^{+}$nerve fibers of serotonin neurons in various brain regions, as previously described (10-12). Among them, nuclei regulating REM sleep and atonia, including the ventrolateral periaqueductal gray (vlPAG), laterodorsal tegmental nucleus (LDTg), and sublaterodorsal nucleus (SLD), also known as the nucleus pontis oralis, peri-LC $\alpha$, and subcoeruleus (25-29), received relatively dense $\mathrm{EYFP}^{+}$projections. We also detected prominent staining in the substantia nigra compact part ( $\mathrm{SNc}$ ) and amygdala, especially in the basolateral nucleus of the amygdala (BLA), as shown in Fig. $3 A$ and Fig. S3A (8). The SNc plays a critical role in controlling movement and has also been implicated in regulating REM sleep and cataplexy 
A
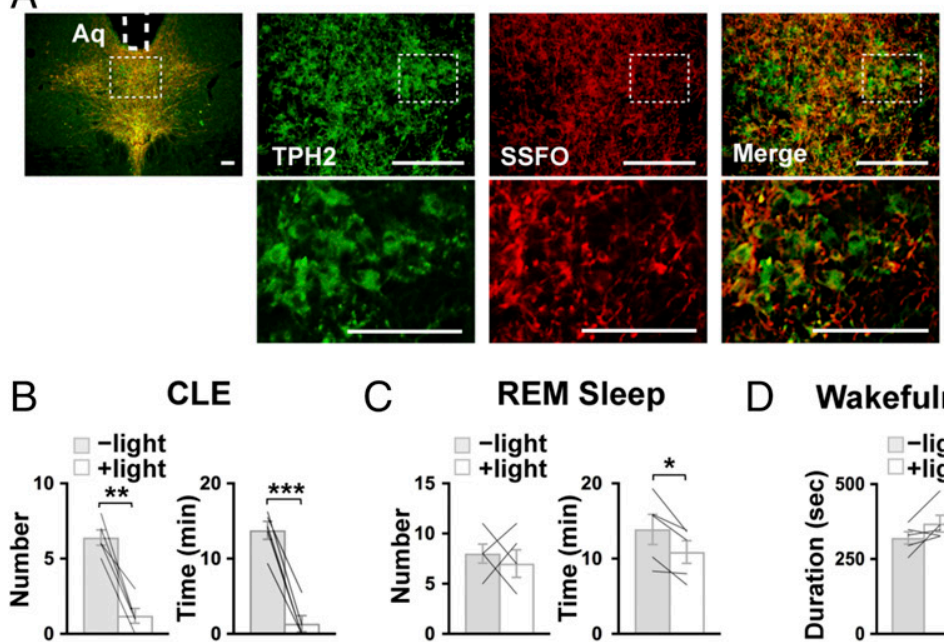

C

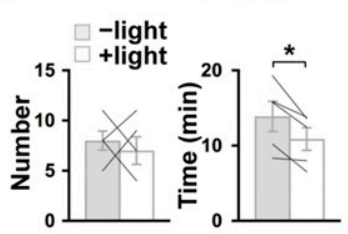

D Wakefulness

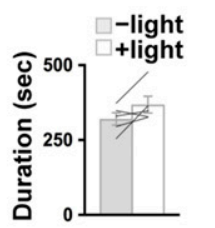

E
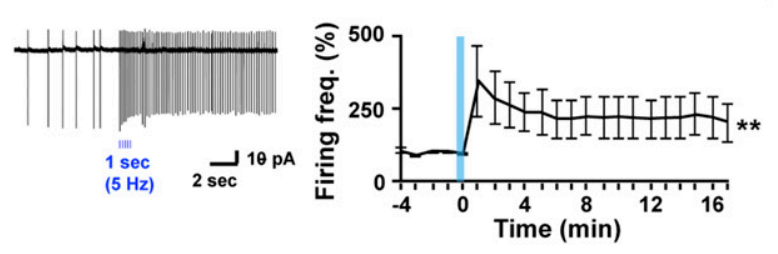

F DRN SSFO(-) +light
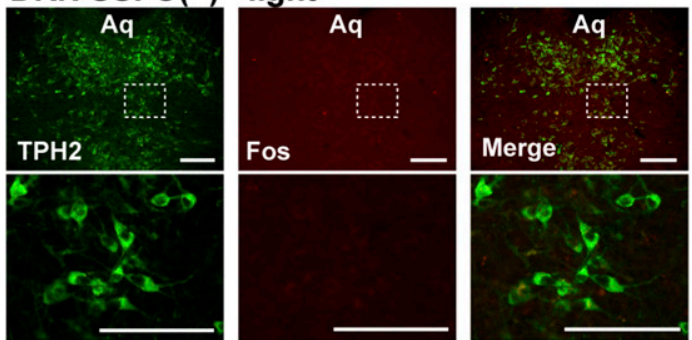

DRN SSFO(+) +light


Fig. 2. Optogenetic activation of DRN serotonergic neurons inhibits CLEs in orexin-ataxin3 mice. (A) Coronal brain sections containing the DRN prepared from Sert-Cre;orexin-ataxin3 mice with a targeted injection of AAV-EF1 $\alpha$-DIO-SSFO-EYFP in the DRN were double-stained with anti-TPH (green) and anti-GFP (red) antibodies. Regions outlined with dashed lines are shown at higher magnification. The position of an optical fiber implant is indicated by a dotted white line. $(B-D)$ Episode number of and time spent in CLE $(B)$, REM sleep $(C)$, or mean duration of wakefulness episodes $(D)$ during $4 \mathrm{~h}$ of chocolate feeding at the onset of the dark phase $(n=5)$. CLEs were suppressed by the optogenetic activation of DRN serotonin neurons in Sert-Cre;orexin-ataxin3 mice. ${ }^{*} P<0.05$, $\star * P<0.01, * * * P<0.001$, two-tailed Student's paired $t$ test. ( $E$ ) Photostimulation of SSFO (10-ms pulses of $5 \mathrm{~Hz}$ for $1 \mathrm{~s}$ ) expressed in DRN serotonin neurons increased their firing frequency in the brain slices. A representative trace of loose-patch clamp recording appears on the left and the time course of mean frequency relative to the baseline rate $(3.9 \pm 1.9 \mathrm{~Hz})$ on the right $(n=4)$. ${ }^{*} P<0.01$, effect of time, Friedman's test. $(F)$ Coronal DRN sections were prepared from Sert-Cre;orexin-ataxin3 mice with (SSFO+) or without (SSFO-) the expression of SSFO-EYFP in the DRN after $4 \mathrm{~h}$ of chocolate feeding along with photostimulation at the DRN. Slices were then double-stained with anti-TPH (green) and anti-Fos (red) antibodies. Regions outlined with dashed lines are shown at higher magnification. Fos ${ }^{+} \mathrm{TPH}^{+}$cells are indicated by white arrowheads. $(G)$ The number of Fos-positive $\mathrm{TPH} 2^{+}$cells, expressed as a percentage of total TPH2 ${ }^{+}$cells, increased with the activation of SSFO at the DRN $(n=5)$. Bar graphs show mean \pm SEM, $* \star * P<0.001$, two-tailed Student's $t$ test. (Scale bar, $100 \mu m$.)

$(30,31)$, whereas the amygdala plays an important role in emotional processing (32) and likely involved in cataplexy triggered by strong emotion $(4,19,33)$.

We placed the optic fiber above these areas unilaterally and optogenetically stimulated serotonin projections individually in SertCre;orexin-ataxin3 mice with an AAV-EF1 $\alpha$-DIO-SSFO-EYFP injection in the DRN. Stimulating serotonin terminals in REMregulating nuclei such as the vlPAG, LDTg, SubC, and SNc did not change the frequency of CLE or the mean duration of wakefulness episodes in the mice during chocolate feeding (Fig. 3B). By clear contrast, when serotonin terminals in the amygdala, especially the central amygdala (CeA) and lateral/basolateral amygdala (LA/ BLA), were optogenetically stimulated, CLEs were suppressed almost completely, whereas the mean duration of wakefulness episodes, REM sleep time, and REM sleep latency remained unchanged (Fig. $3 B$ and Tables S2 and S3).

To confirm that the activation of SSFO at nerve terminals indeed increased the release of serotonin, we illuminated the SSFO-expressing DRN serotonin terminals in coronal slices containing the amygdala and its surrounding areas. Blue-light stimulation clearly increased the amount of serotonin in the extracellular solution, thereby suggesting that SSFO activation in serotonin nerve terminals enhanced the synaptic release of serotonin in the amygdala and its adjacent areas (Fig. 4A).

Because strong emotion often triggers cataplexy, we speculated that DRN serotonin terminals in the amygdala reduce amygdala activity as a means of inhibiting CLEs. As expected, the optogenetic activation of amygdala serotonin terminals significantly reduced Fos protein expression in both the CeA and LA/BLA (Fig. $4 B$ and $C$ ). We also examined amygdala activity following the optic stimulation of SSFO-expressing serotonin neurons in the DRN. The optogenetic excitation of cell bodies of those neurons showed significantly reduced Fos protein expression in the CeA and LA/BLA (Fig. $4 D$ ), thereby recapitulating the optogenetic stimulation of serotonin projections in the amygdala (Fig. $4 C$ ). Such results suggest that SSFO activation in serotonin nerve terminals indeed increased the synaptic release of serotonin and inhibited amygdala activity. Using slice electrophysiology, we also confirmed that serotonin release from the terminals of DRN neurons indeed inhibits BLA principal neurons (see, for example, Fig. $6 F$ and $G$ ).

Chemogenetic Manipulations of Amygdala Activity Bidirectionally Modulate the Occurrence of CLE in orexin-ataxin3 Mice. To further confirm the importance of the inhibitory action on amygdala activity in suppressing CLEs, we expressed an inhibitory DREADD in the amygdala of orexin-ataxin3 mice by bilaterally microinjecting a pair of AAV vectors: AAV-EF1 $\alpha$-DIO-hM4Di-mCherry and AAV-SynI$i C r e$, which expresses codon-improved Cre (iCre) (34) specifically in neurons. hM4Di is a DREADD receptor that induces neuronal hyperpolarization upon binding with its ligand, clozapine- $N$-oxide (CNO) (24). 
A Amygdala
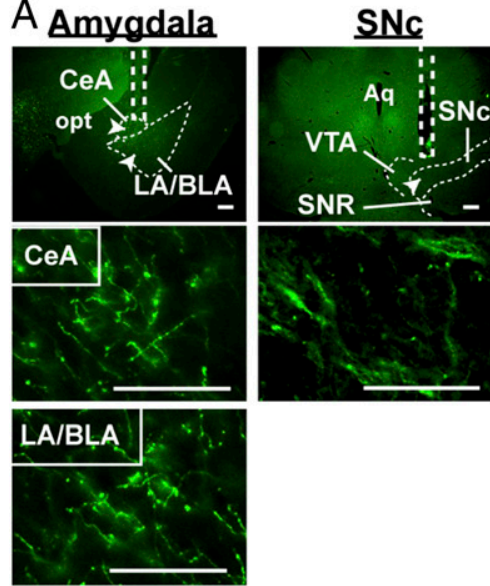
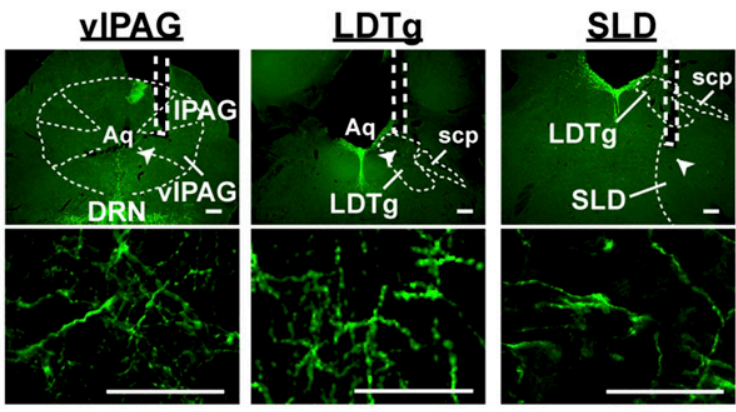

(1)
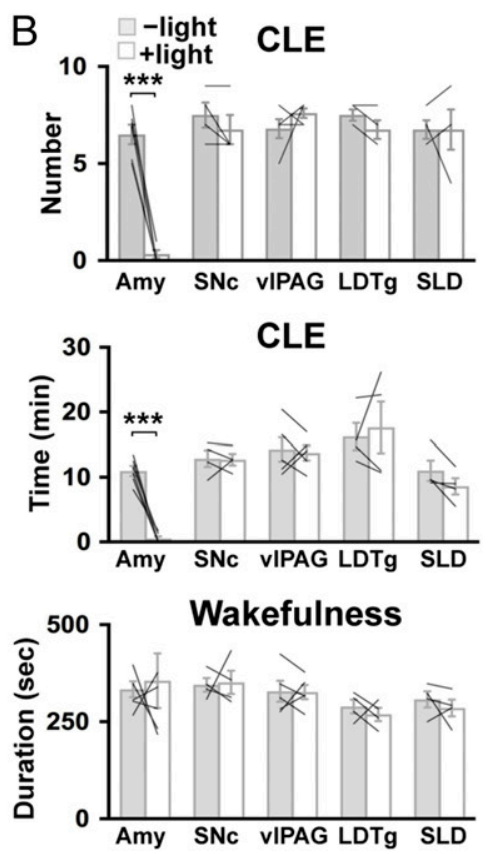

Fig. 3. Optogenetic stimulation of DRN serotonin projections in the amygdala inhibits CLEs in orexin-ataxin3 mice. (A) Representative coronal brain sections of the amygdala, SNc, vIPAG, LDTg, and SLD prepared from Sert-Cre;orexin-ataxin3 mice with targeted injection of AAV-EF1 $\alpha$-DIO-SSFO-EYFP in the DRN. Samples show relatively dense projections of DRN serotonin neurons and the optical fiber tracks. SSFO-EYFP ${ }^{+}$fibers were stained with anti-GFP antibody (green). Regions denoted by white arrowheads are shown at higher magnification. IPAG, lateral periaqueductal gray; opt, optic tract; scp, superior cerebellar peduncle; SNR, substantia nigra reticular part. (Scale bars, $100 \mu \mathrm{m}$.) (B) Sert-Cre;orexin-ataxin3 mice expressing SSFO in DRN serotonergic neurons were unilaterally photostimulated in the regions indicated. The graphs show the episode number of and time spent in CLE, as well as mean duration of wakefulness episodes during a 4-h chocolate-feeding period with (+light) or without (-light) photostimulation (10-ms pulses of $5 \mathrm{~Hz}$ for $1 \mathrm{~s}$, delivered every 30 min). Photostimulation in the amygdala (Amy) specifically inhibited CLEs. $n=5$ for the amygdala and vIPAG; $n=4$ for the SNc, LDTg, and SLD. Bar graphs show mean \pm SEM, ${ }^{* * *} P<0.001$, two-tailed Student's paired $t$ test.

In the amygdala of orexin-ataxin3 mice that received an AAV microinjection, hM4Di-mCherry was expressed in the vast majority of the area covering the CeA $(98.5 \pm 1.0 \%)$ and LA/BLA $(89.6 \pm$ $5.3 \%$ ), as estimated by immunostaining with an anti-mCherry antibody (Fig. $5 A$ and Fig. S $4 D$ ). Immediately before commencing the dark phase, we administrated saline or CNO intraperitoneally in orexin-ataxin3 mice expressing hM4Di in the amygdala and recorded EEG/EMG for the subsequent $12 \mathrm{~h}$ without chocolate feeding, as performed in our previous study (8). Compared with saline, CNO substantially reduced the frequency of and time spent in CLEs during the dark phase (Fig. $5 B$ and Table S4). In contrast, the fragmentation of wakefulness did not improve. Interestingly, $\mathrm{CNO}$ administration did not reduce CLEs in mice with hM4DimCherry expression in the CeA but not in the LA/BLA $(81.4 \pm$ $2.5 \%$ of CeA, $15.0 \pm 6.0 \%$ of LA/BLA) (Fig. S3 $B$ and $C$ and Table S4). Such results suggest that reducing the activity of LA/BLA might be more critical than that of CeA in suppressing CLEs.

In a complementary experiment, we expressed an excitatory DREADD, hM3Dq (24), in the amygdala of orexin-ataxin3 mice to artificially increase amygdala activity. To that end, we bilaterally microinjected a pair of AAV vectors: AAV-EF1 $\alpha$-DIO$h M 3 D q-m C h e r r y$ and AAV-SynI-iCre. In the amygdala of orexinataxin3 mice receiving an AAV microinjection, hM3Dq-mCherry was expressed in the vast majority of the area covering the CeA $(92.6 \pm 4.3 \%)$ and LA/BLA $(92.0 \pm 4.0 \%)$, as estimated by immunostaining with anti-mCherry antibody (Fig. $5 C$ and Fig. $\mathrm{S} 4 D$ ). Compared with saline, $\mathrm{CNO}$ administration significantly increased the frequency of and time spent in CLEs during the dark phase (Fig. 5D and Table S4), although the EEG of CLE in mice administered $\mathrm{CNO}$ was indistinguishable from that in mice administered saline (Fig. S2C). In contrast, the fragmentation of wakefulness was not exacerbated. Therefore, the induction of CLE could depend on the level of amygdala activity.

Restoring Orexin Receptors in the Amygdala of $0 \times 1 r^{-1-} 0 \times 2 r^{-/-}$Mice Cannot Suppress CLEs. Because the amygdala weakly expresses orexin receptors and receives some orexin-positive fibers sporadically $(35,36)$, the direct orexinergic regulation of the amygdala in preventing CLEs is more parsimonious, which we did not test in our previous report (8). In response, we examined whether restoring orexin receptor expression in $O x 1 r^{-1-} O x 2 r^{-1-}$ mice blocked CLEs. In the amygdala of $O \times 1 r^{-1-} O \times 2 r^{-1-}$ mice that bilaterally received an AAV microinjection-a mixture of the recombinant AAV vectors AAV-EF1a-Ox1r-EGFP and AAVEF1a-Ox2r-EGFP (8)-orexin receptors were expressed in the vast majority of the area covering the $\mathrm{CeA}(85.4 \pm 9.5 \%)$ and LA/BLA $(93.6 \pm 4.2 \%)$, as estimated by immunostaining with an anti-GFP antibody (Fig. S5). However, mice with such restoration have demonstrated frequent CLEs (8) in conditions with or without chocolate feeding (Table S5). Because those results suggest that endogenous orexin release in the amygdala cannot alone prevent CLEs, suppressing cataplexy in orexin-deficient mice with the ectopic overexpression of orexin peptides in the amygdala is clinically relevant but perhaps not physiological (37). Similar to our current findings, results of other studies, including our own, have shown that orexin neurons regulate the amygdala indirectly via LC noradrenergic neurons as part of modulating fear learning $(38,39)$.

CLE Relapses When DRN Projection to the Amygdala Is Optogenetically Inhibited in $0 \times 1 r^{-1-} 0 \times 2 r^{-/-}$Mice with OX2R Restoration in DRN Serotonin Neurons. Finally, we aimed to demonstrate the involvement of the DRN-amygdala pathway in the orexinergic suppression of CLEs. We previously showed that the selective 
A

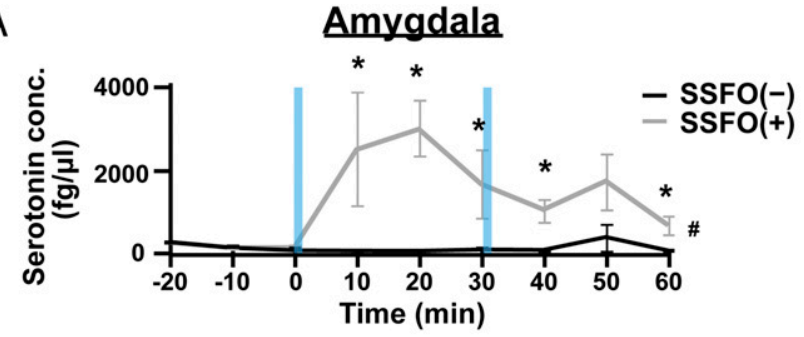

B Amygdala SSFO(-) +light
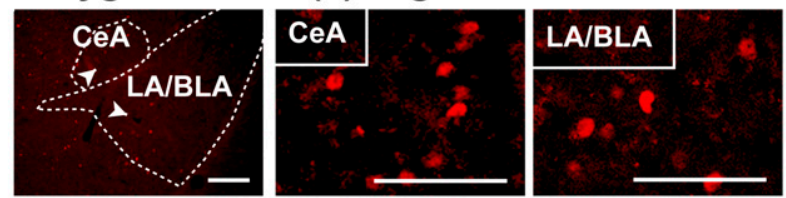

Amygdala SSFO(+) +light


Amygdala stimulation

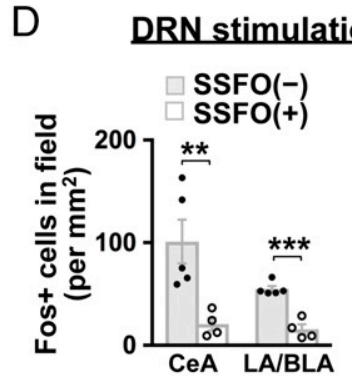

Fig. 4. SSFO activation in serotonin nerve terminals increases serotonin release and inhibits the activity of amygdala in orexin-ataxin3 mice. (A) Coronal slices containing the amygdala were photostimulated $(5 \mathrm{~Hz}, 1 \mathrm{~s})$ at the time indicated by blue columns. Serotonin concentrations in the extracellular solution are plotted. $n=4$ for SSFO(-); $n=5$ for SSFO(+). The pound symbol $\left({ }^{\#}\right)$ represents interaction between time and SSFO, $P<0.05$, two-way repeated-measures ANOVA; The asterisk (*) represents SSFO(-) versus SSFO(+), $P<0.05$, Mann-Whitney's $U$ test. $(B)$ Coronal amygdala sections were prepared from Sert-Cre;orexin-ataxin3 mice with (SSFO+) or without (SSFO-) the expression of SSFO-EYFP in DRN serotonin neurons after $4 \mathrm{~h}$ of chocolate feeding along with photostimulation at the amygdala. Slices were then immunostained with anti-Fos (red) antibody. Regions denoted by white arrowheads are shown at higher magnification. (Scale bars, $100 \mu \mathrm{m}$.) (C) The number of Fos ${ }^{+}$cells was reduced in both the CeA and LA/BLA by the optogenetic activation of DRN serotonin nerve terminals at the amygdala $(n=5)$. (D) The number of Fos ${ }^{+}$cells was reduced in both the CeA and LA/BLA by the optogenetic activation of DRN serotonin cell bodies at the DRN $(n=5)$. Bar graphs show the mean $\pm \mathrm{SEM}, * * P<0.01, * * * P<0.001$, two-tailed Student's $t$ test.

restoration of OX2R expression in DRN serotonergic neurons suppressed CLEs, but not the fragmentation of wakefulness, in narcoleptic $O \times 1 r^{-1-} O \times 2 r^{-1-}$ mice lacking both orexin receptor subtypes (8). Therefore, we examined whether the inhibition of serotonin presynaptic terminals in the amygdala block the anticataplectic effects of OX2R restoration in DRN serotonin neurons. To that end, we expressed the vertebrate short-wavelength

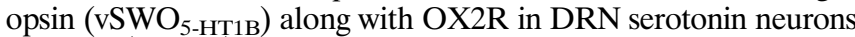
of $O \times 1 r^{-1-} O \times 2 r^{-1-}$ mice by microinjecting a mixture of three recombinant AAV vectors in the DRN: AAV-Pet1-Ox2r-EYFP, AAVEF1 $\alpha-D I O-v S W O_{5-H T 1 B}-m C h e r r y$, and AAV-SynI-iCre. $\mathrm{vSWO}_{5-\mathrm{HT} 1 \mathrm{~B}}$ is a mouse short-wavelength opsin fused with the $\mathrm{C}$ terminus of the
5- $\mathrm{HT}_{1 \mathrm{~B}}$ receptor at its own $\mathrm{C}$ terminus that induces neuronal inhibition by activating $G_{i / o}$ signaling pathways upon stimulation with blue light (40). In those mice, OX2R-EYFP was expressed in $81.2 \pm 1.13 \%$ of $\mathrm{TPH}^{+}$cells, and $98.96 \pm 1.04 \%$ of $\mathrm{EYFP}^{+}$cells were $\mathrm{TPH}_{2}^{+}$, whereas $\mathrm{vSWO}_{5-\mathrm{HT} 1 \mathrm{~B}^{-}}$Cherry was expressed in $83.1 \pm 3.89 \%$ of $\mathrm{TPH}^{+}$cells, and $80.20 \pm 5.18 \%$ of mCherry $^{+}$ cells were TPH $2^{+}(n=7)$, as shown in Fig. $6 A$. We also observed mCherry $^{+}$nerve fibers in the amygdala (Fig. $6 B$ ).

Without optogenetic stimulation, $O \times 1 r^{-1-} O x 2 r^{-/-}$mice with OX2R and vSWO $_{5-\mathrm{HT} 1 \mathrm{~B}}$ expression in their DRN serotonin neurons demonstrated few CLEs during the chocolate-feeding period at the beginning of dark phase, as consistent with the results of our previous study (8). By clear contrast, bilaterally applying blue light in the amygdala $(473 \mathrm{~nm}$, for 5 min every 30 min) (Fig. S1B) induced numerous CLEs in the same mice (Fig. 6C and Table S6). Such results suggest that the DRN serotonin neuron-amygdala pathway is critical in suppressing CLE via orexin neurons.

A



hM4Di
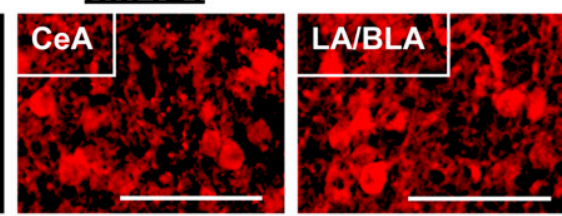

B

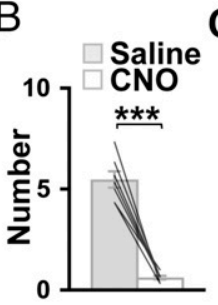

CLE

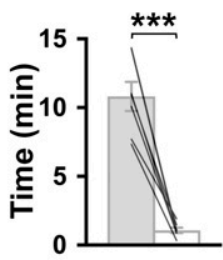

Wakefulness

C

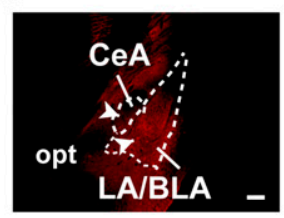

$h M 3 D q$
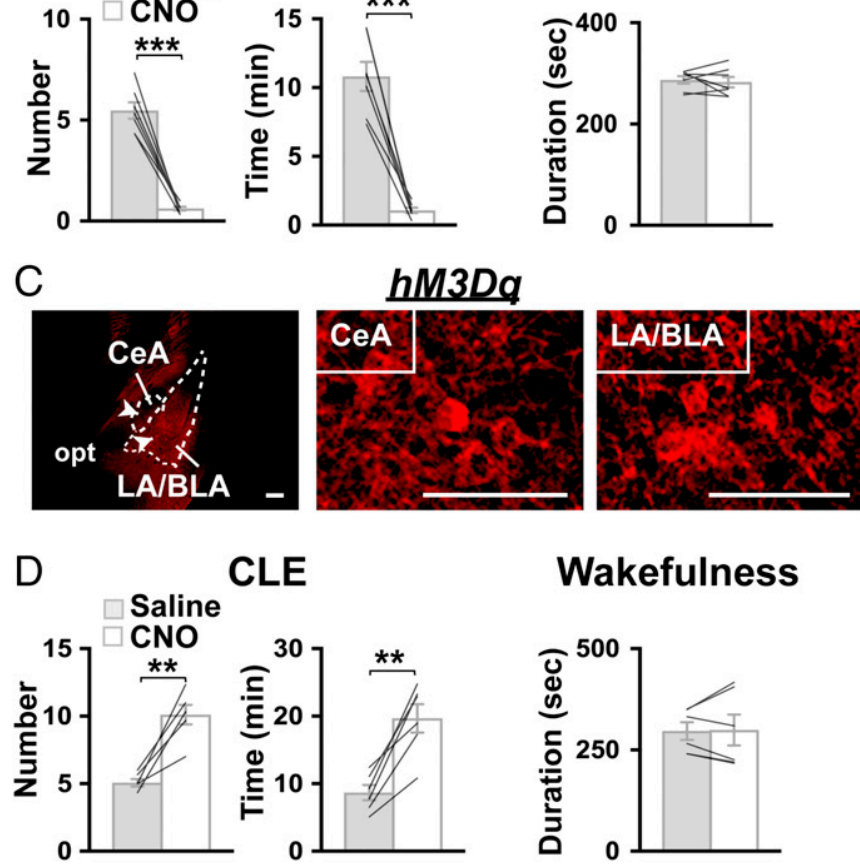

CLE

Wakefulness

Fig. 5. Chemogenetic manipulations of amygdala activity bidirectionally changes the frequency of CLE in orexin-ataxin3 mice. ( $A$ and $C$ ) Coronal brain sections of the amygdala prepared from orexin-ataxin3 mice with bilateral injection of AAV-Synl-iCre and AAV-EF1 $\alpha$-DIO-hM4Di-mCherry $(A)$ or AAV-EF1 $\alpha$-DIO-hM3Dq-mCherry (C) in the amygdala (CeA and LA/BLA) were stained with anti-mCherry (red) antibody. Regions denoted by white arrowheads are shown at higher magnification. (Scale bars, $100 \mu \mathrm{m}$.) $(B$ and $D)$ Orexin-ataxin3 mice with hM4Di-mCherry $(B)$ or hM3Dq-mCherry $(D)$ expression in the amygdala were injected with saline or CNO at the onset of the dark phase. The graphs show episode number of and time spent in CLE; mean duration of wakefulness episodes during the 12-h dark phase without chocolate feeding are also shown ( $n=7$ for hM4Di, $n=4$ for hM3Dq). Bar graphs show mean \pm SEM, $* * P<0.01$, $* * * P<0.001$, two-tailed Student's paired $t$ test. 
ADRN
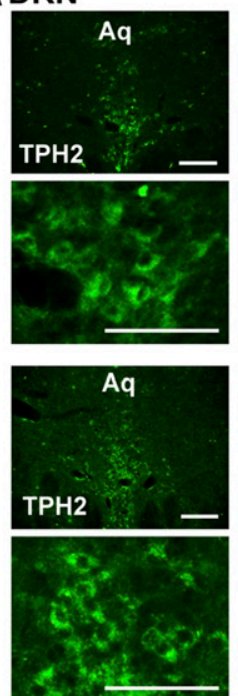
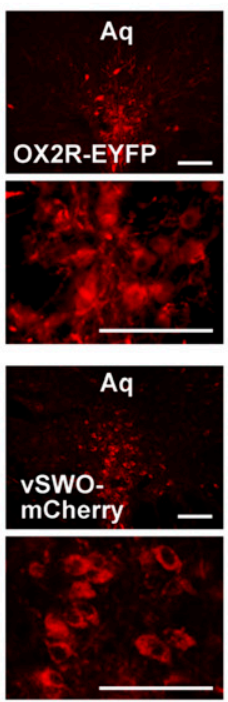
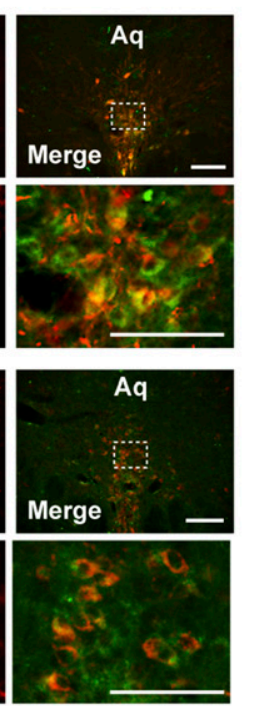

B Amygdala
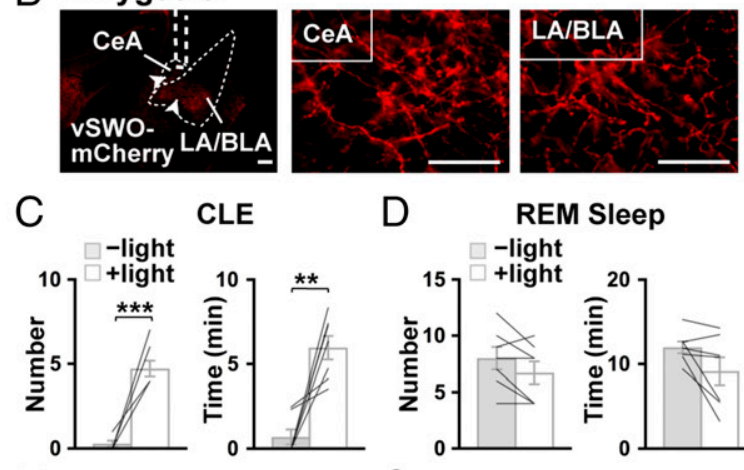

D

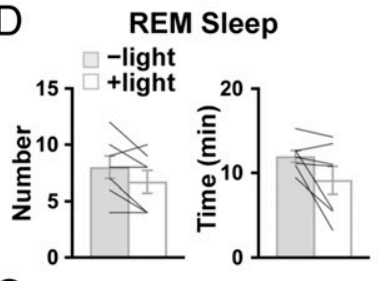

$\mathrm{F}$

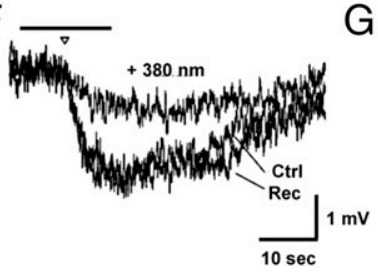

$\mathrm{G}$

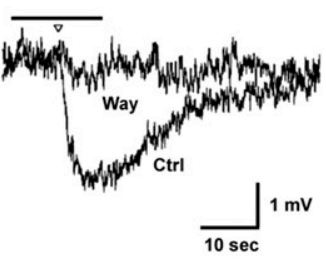

E Wakefulness

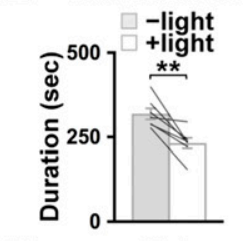

$\mathrm{H}$

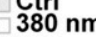

Rec

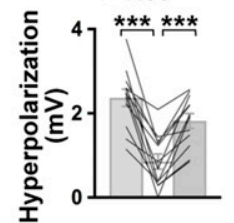

Fig. 6. Restoration of OX2R in DRN serotonin neurons fails to suppress CLE in $O \times 1 r^{-1-} O \times 2 r^{-1-}$ mice when DRN projections in the amygdala are optogenetically inhibited. (A) Coronal brain sections containing the DRN prepared from Ox1 $r^{-1-} O \times 2 r^{-1-}$ mice with the targeted injection of AAV-Pet1-Ox2r-EYFP, AAV-EF1 $\alpha$-DIO-vSWO ${ }_{5-H T 18}$ - $m$ Cherry and AAV-Synl-iCre in the DRN were double-stained with anti-TPH (green) and anti-GFP (red) antibodies or anti-TPH (green) and anti-mCherry (red) antibodies. Regions outlined by dashed lines are shown at higher magnification. (B) Coronal brain sections containing the amygdala were stained with anti-mCherry antibody (red). The position of an optical fiber implant is indicated by dotted white line. Regions denoted by white arrowheads are shown at higher magnification. (Scale bars, $100 \mu \mathrm{m}$.) (C-E) Episode number of and time spent in CLE (C), REM sleep (D), or mean duration of wakefulness episodes $(E)$ during $4 \mathrm{~h}$ of chocolate feeding at the onset of the dark phase $(n=5)$. CLE relapsed when blue light was applied (+light) in the

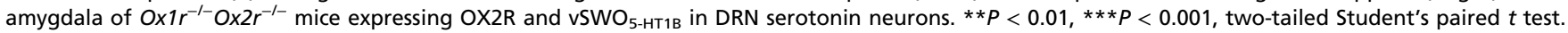
$(F$ and $G)$ In the amygdala principal neurons, slowly developing hyperpolarization was evoked by the photostimulation of hChR2-expressing serotonin fibers with a train of $10473-\mathrm{nm}$ light flashes $(10 \mathrm{~Hz})$, each lasting $10 \mathrm{~ms}$, delivered at the timing pointed on the traces (triangle). The photo-evoked hyperpolarization was reduced by a preceding 380 -nm light illumination ( $16 \mathrm{~s}$, shown by a line) $8 \mathrm{~s}$ before the $473-\mathrm{nm}$ light train $(n=12$ cells) $(F)$ and abolished with the application of 5-HT1A receptor antagonist, WAY $100635(30 \mu \mathrm{M})(n=2$ cells) $(G)$. (H) Summary bars showing changes in the amplitude of the 473-nm lightevoked hyperpolarization by the preceding $380 \mathrm{~nm}$ light. Hyperpolarization was recovered without the 380-nm light (Rec). Bar graphs show mean \pm SEM, $* * * P<0.001$, one-way repeated-measures ANOVA followed by Tukey's test.

We also confirmed that $\mathrm{vSWO}_{5-\mathrm{HT} 1 \mathrm{~B}}$ stimulation can reduce serotonin release from the nerve terminals in the amygdala via electrophysiological recordings and ChR2-assisted transmitter release in brain slice preparation (Fig. $6 F-H)$. To do that, we expressed hChR2(H134R) in serotonin neurons of the DRN along with $\mathrm{vSWO}_{5-\mathrm{HT} 1 \mathrm{~B}}$ by microinjecting AAV-EF1 $\alpha-D I O-$ $\nu S W O_{5-H T 1 B}-m C h e r r y$ and AAV-EF1 $\alpha$-DIO-hChR2(H134R)-EYFP in Sert-Cre mice. In neurons of the BLA, presumably principal neurons according to their size, slowly developing hyperpolarization was evoked by a train of 473-nm light flashes delivered via an optical fiber placed near the recording area in the presence of glutamatergic and GABAergic receptor antagonists (Fig. $6 F$ and $H$ ). Hyperpolarization was abolished with the application of a 5-HT1A receptor antagonist, WAY $100635(30 \mu \mathrm{M})$, thereby suggesting that serotonin was released from the terminal in the amygdala and induced the hyperpolarization via 5-HT1A receptors (Fig. 6G). When 380-nm light illumination preceded the blue-light train to activate $\mathrm{vSWO}_{5-\mathrm{HT} 1 \mathrm{~B}}$, blue light-evoked hyperpolarization reduced significantly (Fig. $6 F$ and $H$ ). Such results suggest that serotonin release from DRN neuronal terminals inhibited LA/BLA principal neurons, an action that was reduced by the activation of $\mathrm{vSWO}_{5-\mathrm{HT} 1 \mathrm{~B}}$ in the terminals.

\section{Discussion}

Our study provides additional support for our previous observations that DRN serotonin neurons mediate the anticataplectic function of orexin neurons (8). Both studies propose the orexin neuron-DRN serotonin neuron-amygdala pathway as a critical circuit to prevent cataplexy.
Absence of Anticataplectic Effects of Serotonin Nerve Stimulations in Brainstem Nuclei Involved in Regulating REM Sleep and REM Atonia. Although we failed to suppress CLEs by optogenetically stimulating DRN serotonin neuron terminals in several brain regions involved in regulating REM sleep and REM atonia, including the vlPAG, SLD, LDTg, and SNc (25-31), such results do not completely exclude the contribution of serotonin signaling in those regions in preventing CLEs. Indeed, we have no means of knowing whether the conditions of optogenetic stimulations used in our study were optimal for activating the serotonin projections of interest. Nevertheless, our results indicate that the amygdala was the most efficient downstream target of DRN serotonin neurons in inhibiting CLEs.

The SLD induces atonia during REM sleep by exciting interneurons in the medulla and spinal cord that inhibit motoneurons, whereas during wakefulness, the SLD is inhibited by neurons in the vlPAG and adjacent lateral pontine tegmentum (LPT) (25, 27, 28). Containing REM-on cholinergic neurons, the LDTg has been suggested, albeit not without controversy, to play an important role in inducing REM sleep and REM atonia by inhibiting pontine monoaminergic neurons and activating SLD neurons (26, 29). Dopamine neurons in the SNc play a critical role in fine motor control. They were also implicated in regulating REM sleep and cataplexy, and modulation of their activity via a $\mathrm{D}_{2} / \mathrm{D}_{3}$ agonist and antagonist aggravated and reduced cataplexy, respectively, in narcoleptic dogs $(30,31)$.

Amygdala as the Target Site of DRN Serotonin Neurons in Suppression of CLEs. In narcoleptic patients, cataplexy is most often triggered by positive emotions, such as those associated with laughter, joking, or 
delight (4), as also demonstrated in narcoleptic dogs and mice $(6,7$, 19, 41-43). Unsurprisingly, the serotonergic inhibition of the amygdala prevents CLEs, for the amygdala plays an important role in emotional processing (32), within which LA/BLA subdivisions form the primary input nuclei and receive processed sensory and executive information from cortical and subcortical areas. The LA/ BLA consists of glutamatergic principal neurons and GABAergic interneurons; the former projects to the $\mathrm{CeA}$. The $\mathrm{CeA}$ is generally thought to be an output structure for fear responses (32).

In narcoleptic dogs, a population of sleep-active cells localized to the $\mathrm{CeA}$ and BLA was reported to increase discharges before and during cataplexy (44). Similarly, in human narcolepsy type 1 , amygdala activity increases during cataplexy $(45,46)$. In another study, all REM-active neurons recorded from the CeA were inhibited by DRN stimulation in rats (47). Recently, Oishi et al. demonstrated that Fos expression in the BLA and basomedial amygdala increases after cataplexy-inducing chocolate feeding in narcoleptic orexin $^{-1-}$ mice (19). In an accompanying paper, Burgess et al. reported that excitotoxic lesions of the amygdala markedly reduced cataplexy in orexin $^{-/}$mice without altering basic sleep/wakefulness (33).

GABAergic CeA neurons send dense innervation to the vlPAG/LPT, which inhibits REM sleep and maintains waking muscle tone $(33,48,49)$. At the same time, the SLD receives glutamatergic innervation from the CeA (50-52). Taking these data together, we find the amygdala is anatomically well positioned to regulate muscle tone and REM sleep (28).

In the LA/BLA of amygdala slices, serotonin produces a primarily inhibitory response by increasing the GABAergic transmission of interneurons, which subsequently inhibits principal excitatory neurons $(53,54)$, and directly hyperpolarizes a minority of principle neurons $(53,55)$. We also observed its direct inhibition in the current study (Fig. 6). Although we did not discriminate direct and indirect actions of serotonin on the principal neurons, such electrophysiological findings are consistent with our results that the optogenetic stimulation of serotonin terminals reduces Fos expression in the LA/BLA, where glutamatergic principal neurons predominate.

As mentioned earlier, cataplexy is usually triggered by positive emotions, yet rarely by negative ones, such as fear and anxiety (4). Indeed, studies have shown abnormal amygdala responses to emotional stimuli in narcolepsy patients, including increased responses to positive rewards and decreased ones to aversive stimuli $(4,56-58)$. Because serotonin transmission in the LA/ BLA participates in the acquisition of cued conditioned fear (59), we cannot exclude the possibility that the optogenetic stimulation of serotonin terminals in the LA/BLA suppressed CLEs by producing fear. In any case, it should be noted that the involvement of LA/BLA serotonin transmission in the production of fear remains controversial (59-65).

Although emotional processing in the amygdala has been studied extensively regarding the expression and learning of fear, several lines of evidence have also suggested that it is critically involved in processing rewarding stimuli $(32,66-73)$. Different populations of LA/BLA neurons likely encode fearful or rewarding information $(70,71,74,75)$. In contrast to the circuit for fear, output from the LA/BLA mediating positive valence could be independent of the $\mathrm{CeA}$ and depend on its projections to other brain regions, including the nucleus accumbens (NAc), which mediates motivated response to reward-predictive cues for both natural and drug rewards $(66,75)$. Notably, inhibiting CeA activity did not reduce CLEs in our study (Fig. S3C). Other research has demonstrated that the activation of LA/BLA $5-\mathrm{HT}_{2 \mathrm{~A} / \mathrm{C}}$ receptors inhibits behaviors related to reward seeking by suppressing the LA/BLA principal neuronal activity (76), and that chocolate feeding induces Fos in the BLA more than in the CeA of orexin $^{-1-}$ mice (19). The LA/BLA could thus be a site that plays a critical role in triggering cataplexy by processing environmental stimuli that provoke positive emotions.

Orexin neurons respond to drug cues and mediate drug seeking via projections to the dopaminergic ventral tegmental area (VTA), as studies with rodents have demonstrated (77-79). During high reward expectancy, narcolepsy type 1 patients did not show increased VTA activity as controls did, but instead exhibited abnormally increased activity in the amygdala $(4,56)$. Taken in conjunction with our current results, given rewarding stimuli, orexin neurons might activate the VTA to increase dopamine efflux. At the same time, orexin neurons could also activate DRN serotonin neurons to reduce amygdala response. In all, the amygdala is likely to carry the emotional aspect of rewards.

A Model for How Orexin Neurons Suppress Cataplexy. Because cataplexy and the atonia of REM sleep share many characteristics, they are likely to be mediated by the same brainstem pathways. The model derived from previous studies postulates the orexinergic augmentation of vlPAG/LPT directly or indirectly, if not both, via pontine monoaminergic neurons to counterbalance cataplexy-triggering signals from the $\mathrm{CeA}$, which inhibit the vlPAG/LPT or stimulate the SLD, if not both $(4,19,28,33)$.

We propose a neuronal pathway by which orexin neurons exert their anticataplectic action via DRN serotonin neurons by reducing amygdala activity, probably at the LA/BLA (Fig. S6). Thus, an orexin neuron-DRN serotonin neuron pathway might inhibit cataplexy by preventing exaggeration of the output of the amygdala, instead of by directly acting on neurons involved in regulating muscle tone. That hypothesis is consistent with the idea that REM sleep and cataplexy are regulated by at least partially distinct mechanisms, as suggested by previous reports and the fact that human narcolepsy patients usually remain conscious during cataplectic attacks $(4,31)$. Indeed, unlike CLE, optogenetic stimulations of serotonin terminals in the amygdala did not improve excessive REM sleep duration, as well as shortened REM sleep latency in orexin-ataxin3 mice (Table S2). Both our previous study (8) and another showing no change in REM sleep by amygdala ablation (33) support that idea.

Technical Considerations. We used a ChR2 variant, SSFO, to optogenetically stimulate DRN serotonin neurons (21). Unlike stimulation with conventional ChR2 variants, optical stimulation of SSFO causes prolonged depolarization and does not pace neuronal firing (20). As such, the action of SSFO in the nerve terminals remained unclear. At the opposite extreme, it could generate a stable increase in calcium that might disrupt the normal function of the terminals, thereby resulting in a paradoxical decrease, not increase, of the neurotransmitter release (20). Nevertheless, we concluded that the SSFO activation enhanced the presynaptic release of serotonin in the amygdala for three reasons. First, the activation of SSFO at serotonin fibers in the amygdala increased the release of serotonin in slices, as observed by direct measurements of extracellular serotonin. Second, changes in Fos expression in the amygdala were similar between the SSFOmediated activation of serotonin neurons in cell bodies in the DRN and at the terminals in the amygdala. Third and lastly, SSFO activates serotonin neuronal terminals in the amygdala and in cell bodies and suppressed CLEs at both sites.

The photoactivation of one axonal projection using conventional ChR2 would be expected to activate the entire neuron, including any putative axon collaterals, via a back-propagating action potential (20). However, the absence of anticataplectic effects of SSFO activation at serotonin neuronal terminals in the vlPAG, SLD, LDTg, and SNc made such an effect unlikely.

We have demonstrated the critical role of the DRN-amygdala pathway in the orexinergic suppression of CLEs by using orexin receptor-deficient mice with a restoration of OX2R expression selectively in DRN serotonin neurons (Fig. 6). Therefore, and as 
discussed in our previous study (8), we cannot exclude the possibility that orexins normally suppress CLEs via additional pathways independent of the DRN-amygdala pathway, because we have failed to induce CLEs in wild-type mice by inhibiting the orexin neuron-DRN serotonin neuron-amygdala pathway. However, demonstrating that effect would be difficult because the pathway should be blocked almost completely $(<5 \%)$ to induce cataplexy, according to Tabuchi et al. (80). Our experiments, the results of which appear in Fig. 6, might overcome such difficulty because the coinjection of $O \times 2 r$-expressing and $v S W O$ expressing AAVs was likely to achieve the coexpression of $\mathrm{OX} 2 \mathrm{R}$ and vSWO in a majority of infected cells. Furthermore, the loss-offunction analyses, including those concerning optogenetic inhibition, sometimes underestimate the importance of the blocked component because of compensation or redundancy, if not both. It should be noted that DRN serotonin neurons are currently the sole direct targets of orexin neurons identified for the efficient suppression of CLEs (8). Therefore, our study has substantial scientific importance in delineating a neuronal circuit for preventing cataplexy.

\section{Materials and Methods}

Animals. Orexin-ataxin3, Ox $1 r^{-1-} \mathrm{O} \times 2 r^{-1-}$, and Sert-Cre (provided by Xiaoxi Zhuang, The University of Chicago, Chicago) mice were described previously $(7,8,22)$. The 12 - to 20 -wk-old male mice (backcrossed to C57BL/6J) were used. Mice were maintained under a strict 12-h light/12-h dark cycle in a temperature- and humidity-controlled room and fed ad libitum. All experimental procedures involving animals were approved by the appropriate institutional animal care and use committee of Kanazawa University.

Generation of Recombinant AAV Vectors. The plasmid $p A A V-E F 1 \alpha-D I O-S S F O$ EYFP-WPRE-pA was provided by Karl Deisseroth, Stanford University, Stanford, CA. This plasmid was modified to construct $p A A V-E F 1 \alpha-D I O-V S W O_{5-H T 1 B^{-}}$ $m$ Cherry-WPRE-pA by replacing SSFO-EYFP with $v^{2} W_{5-H T 1 B}-m C h e r r y ~(40)$ The plasmid $\mathrm{p} A A V-E F 1 \alpha-D I O-h M 4 D i-m C h e r r y-W P R E-p A$ and $\mathrm{pAAV}-E F 1 \alpha-D I O$ $h M 3 D q-m C h e r r y-W P R E-p A$ were provided by Bryan Roth, University of North Carolina, Chapel Hill, NC. The plasmid pAAV-Synl-EYFP-WPRE-pA (provided by Karl Deisseroth) was modified to construct $p A A V-S y n l-i C r e$ by replacing EYFP with iCre (34) (provided by Rolf Sprengel, Max-Planck Institute for Medical Research, Heidelberg, Germany). Recombinant AAV vectors (AAV2-rh10) were produced using a triple-transfection, helper-free method and purified as described previously (81). The titers of recombinant AAV vectors were determined by quantitative PCR: AAV-EF1 $\alpha$-DIO-SSFO-EYFP, $1.9 \times 10^{13}$; AAV-EF1a-DIOhM4Di-mCherry, $1.1 \times 10^{12}$; AAV-EF1 $\alpha$-DIO-hM3Dq-mCherry, $4.2 \times 10^{12}$; AAV-

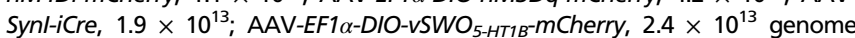
copies per milliliter. AAV vectors AAV-Pet1-Ox2r-EYFP-WPRE-pA, AAV-EF1aOx1r-EGFP, AAV-EF1a-Ox2r-EGFP, and AAV-EF1 $\alpha$-DIO-hChR2(H134R)-EYFP were described previously (8).

Surgery. Implantation of an EEG/EMG electrode, optic fiber, and stereotaxic injection of AAV vectors were performed as described previously (8); $0.5-1.0 \mu \mathrm{L}$ of purified virus was delivered to each site $(0.05 \mu \mathrm{L} / \mathrm{min})$. After $5 \mathrm{~min}$ of rest, the needles were removed. The stereotaxic coordinates of the Hamilton needle syringe placement were as follows (relative to the bregma): DRN (4.4-mm posterior, 0-mm lateral, 3.0- and 3.5-mm ventral); amygdala (1.6-mm posterior, $\pm 2.5-\mathrm{mm}$ lateral, 4.5- and 5.2-mm ventral). The stereotaxic coordinates of the tips of optical fiber implants (BFH48-200, Thorlabs) were as follows: DRN (4.4-mm posterior, 0-mm lateral, 3.0-mm ventral); amygdala (1.6- $\mathrm{mm}$ posterior, $3.0-\mathrm{mm}$ right 4.5-mm ventral); SNc (3.0-mm posterior, $0.8-\mathrm{mm}$ right, $4.2-\mathrm{mm}$ ventral); vIPAG (4.2-mm posterior, $0.5-\mathrm{mm}$ right, $2.5-\mathrm{mm}$ ventral); LDTg $(5.2-\mathrm{mm}$ posterior; $0.6-\mathrm{mm}$ right, $3.1-\mathrm{mm}$ ventral); SLD $(5.2-\mathrm{mm}$ posterior, $1.0-\mathrm{mm}$ right, $4.0-\mathrm{mm}$ ventral). Optical fibers were bilaterally implanted for the $\mathrm{vSWO}_{5-\mathrm{HT} 1 \mathrm{~B}}$ experiment. After surgery, all animals were housed individually for a recovery period of at least $14 \mathrm{~d}$. SSFO(-) control mice were implanted with optical fibers and EEG/EMG electrodes but were not injected with AAV vectors.

After completing EEG/EMG recordings, we evaluated the expression of SSFO-EYFP, hM4Di-mCherry, hM3Dq-mCherry, OX2R-EYFP, vSWO ${ }_{5-\mathrm{HT}^{-} \mathrm{B}^{-}}$ mCherry, OX1R-EGFP, and OX2R-EGFP as described below in recorded mice, before scoring the sleep/wake stages to select those with expression in more than approximately half of the cells of interest.

Feeding with Chocolate. For optogenetic experiments, mice were given milk chocolate after the recovery period (Hershey's Kiss; Hershey) along with their regular chow for $24 \mathrm{~h}$ starting at Zeitgeber time (ZT) 12 (dark phase onset; day 1) (Fig. S1). Four days later (day 5), mice were again fed with chocolate for $4 \mathrm{~h}$ (ZT12-16). On day 9, an EEG/EMG was recorded for $4 \mathrm{~h}$ (ZT12-16) as baseline sleep/wakefulness (-light) from mice fed with chocolate. On day 13, the EEG/EMG was again recorded for $4 \mathrm{~h}$ (ZT12-16) with optogenetic stimulation as described below (+light) from mice fed with chocolate.

Optical Stimulation. Blue light $(473 \mathrm{~nm})$ was generated with a DL-473 laser (Rapp OptoElectronic) and delivered to the animal via an optical fiber $(200 \mu \mathrm{m}$ core, 0.22 numerical aperture, Thorlabs; $12-25 \mathrm{~mW}$ at the tip of optical fiber). This was coupled to the fiber optic implanted on the animals via a zirconia sleeve. A pulse generator (Bioresearch center) was used to generate the light stimulation (10-ms pulses at $5 \mathrm{~Hz}$ for $1 \mathrm{~s}$ or a 5 -min pulse) every $30 \mathrm{~min}$ for $4 \mathrm{~h}$.

Sleep Recordings. EEG/EMG recordings were performed for the first $4 \mathrm{~h}$ during the dark phase in optogenetic experiments or for 12 consecutive $h$ in the dark phase in DREADD experiments. EEG/EMG data were analyzed as previously described (8), and EEG/EMG signals were scored in wakefulness, NREM sleep, REM sleep, or cataplexy. For cataplexy, we previously confirmed that direct transitions from wakefulness ( $>40 \mathrm{~s}$ ) to REM sleep ( $>10 \mathrm{~s}$ ) in EEG/EMG data perfectly correlated with episodes of abrupt immobility in video data (8). Such findings satisfy the criteria of Scammell et al. (9). Because we needed to analyze many animals, we decided to record only EEG/EMG for the rest of the mice. Although we believe that the identified episodes were equivalent to murine cataplexy, we have called the direct transitions from wakefulness to REM sleep CLEs, in reference to Scammell et al. (9). REM sleep latency was calculated as the latency from the onset of sleep (i.e., transition from wakefulness to NREM sleep) to the first episode of REM sleep within the particular sleep episode. Sleep episodes without REM sleep were excluded from the calculation of REM sleep latency. A spectral analysis of the EEG was performed by fast Fourier transform using SleepSign (Kissei Comtec). The analysis yielded power spectra profiles within a 0 - to $20-\mathrm{Hz}$ window with a $1-\mathrm{Hz}$ resolution. Data were standardized by expressing each frequency bin as a percentage relative to the total power of the same epochs.

Drug Administration. The CNO (C0832, Sigma-Aldrich) was dissolved in saline to a concentration of $0.5 \mathrm{mg} / \mathrm{mL}$. The CNO or saline was administered to each mouse intraperitoneally $(0.3 \mathrm{~mL} / 30 \mathrm{~g}$ body weight) at $\mathrm{ZT} 12$, and then EEG/ EMG was recorded for $12 \mathrm{~h}$ (ZT12-0) without chocolate feeding. This dose of CNO has been confirmed to have no off-target effects on sleep and wakefulness (8). Each mouse received three saline and three CNO administrations in an alternating manner at intervals of $1 \mathrm{~d}$ after saline administration or $2 \mathrm{~d}$ after CNO administration.

Histological Study. Double immunostaining was performed as described previously (8). Serial coronal brain sections (30- $\mu \mathrm{m}$ thick) were collected in four series, one of which was further immunostained. The EYFP and mCherry fluorescence was completely quenched before immunostaining by treating sections with $0.3 \%$ hydrogen peroxide. Antibodies used included: rabbit anti-GFP (1:500; Invitrogen); mouse anti-TPH (1:500; Sigma-Aldrich); rabbit anti-cFos (1:15000; sc-52, Santa Cruz Biotechnology); goat anti-mCherry (1:500; SICGEN); Alexa Fluor 488-conjugated donkey anti-mouse IgG (1:1,000; Molecular Probes); Alexa Fluor 594-conjugated donkey anti-rabbit IgG (1:1,000; Molecular Probes). A representative optical section was imaged from each stained section via a fluorescence microscope (BZ-X710; Keyence), and fluorescent cells in the images were counted. In evaluating hM4Di-mCherry or hM3Dq-mCherry expression in the amygdala, it was difficult to count the number of positive cells because the hM4Di-mCherry and hM3Dq-mCherry were expressed at a high level driven by a ubiquitous promoter and were strongly localized in the cell membrane and nerve fibers. Therefore, we measured the areas containing positive cells within the LA/BLA and CeA to obtain rough estimates of expression.

Electrophysiological Recording. Three- to 6-wk-old Sert-Cre;orexin-ataxin3 mice were injected in the DRN with AAV-EF1 $\alpha$-DIO-SSFO-EYFP or a mixture of AAV-EF1 $\alpha$-DIO-hChR2(H134R)-EYFP and AAV-EF1 $\alpha$-DIO-VSWO ${ }_{5-H T 1 B}-m C h e r r y$. After 2-4 wk, the mice were decapitated under deep anesthesia with isoflurane (Mylan). Brains were extracted and cooled in ice-cold cutting solution consisting of $87 \mathrm{mM}$ of $\mathrm{NaCl}, 75 \mathrm{mM}$ of sucrose, $25 \mathrm{mM}$ of $\mathrm{NaHCO}_{3}, 10 \mathrm{mM}$ of D(+)-glucose, $7 \mathrm{mM}$ of $\mathrm{MgCl}_{2}, 2.5 \mathrm{mM}$ of $\mathrm{KCl}, 1.25 \mathrm{mM}$ of $\mathrm{NaH}_{2} \mathrm{PO}_{4}$, and $0.5 \mathrm{mM}$ of $\mathrm{CaCl}_{2}$ bubbled with $\mathrm{O}_{2}(95 \%)$ and $\mathrm{CO}_{2}(5 \%)$ for the DRN recording. Choline chloride (125 mM) replaced the $\mathrm{NaCl}$ and sucrose for the amygdala recording. Coronal brain slices (250- $\mu \mathrm{m}$ thick) including the DRN or amygdala were prepared with a vibratome (VT1200S, Leica) and incubated for $1 \mathrm{~h}$ at room temperature in 
artificial cerebrospinal fluid (ACSF) containing $125 \mathrm{mM}$ of $\mathrm{NaCl}, 26 \mathrm{mM}$ of $\mathrm{NaHCO}_{3}, 10 \mathrm{mM}$ of $\mathrm{D}(+)$-glucose, $2.5 \mathrm{mM}$ of KCl, $2 \mathrm{mM}$ of $\mathrm{CaCl}_{2}$, and $1 \mathrm{mM}$ of $\mathrm{MgSO}_{4}$ bubbled with $\mathrm{O}_{2}(95 \%)$ and $\mathrm{CO}_{2}(5 \%)$. Spontaneous action potentials of SSFO-EYFP-expressing neurons were recorded with loose cell-attached modes at $30^{\circ} \mathrm{C}$ kept by perfusion of preheated ACSF. Recording glass electrodes were filled with ACSF (2-4M $\Omega$ ). A combination of EPC10/2 amplifier and Patchmaster software (HEKA) was used to control membrane voltage, data acquisition, and the triggering of light pulses. A 470-nm LED device (Rapp OptoElectronic) was used for SSFO stimulation ( $35 \mu \mathrm{W}$ ). Before commencing DRN recording, the slices were illuminated with yellow light $(575 \mathrm{~nm}, 10 \mathrm{~mW})$ for $5 \mathrm{~s}$ to inactivate SSFO and obtain the baseline neuronal activity of the targeted cells. In the amygdala principal neurons, whole-cell recordings were made with the electrodes (4-5 $\mathrm{M} \Omega$ ) filled with an internal solution containing $125 \mathrm{mM}$ of K-gluconate, $4 \mathrm{mM}$ of NaCl, $10 \mathrm{mM}$ of Hepes, $0.2 \mathrm{mM}$ of EGTA, $2 \mathrm{mM}$ of $\mathrm{MgCl}_{2}, 4 \mathrm{mM}$ of ATP, $0.4 \mathrm{mM}$ of GTP, and $10 \mathrm{mM}$ of phosphocreatine (pH 7.3, adjusted with $\mathrm{KOH})$. Neurons were current-clamped at room temperature in the presence of bicuculline $(10 \mu \mathrm{M}), 6$-cyano-7-nitroquinoxaline-2,3-dione disodium (CNQX $10 \mu \mathrm{M})$, and D-(-)-2-amino-5-phosphonopentanoic acid (D-AP5, $30 \mu \mathrm{M}$; TOCRIS) to record light-evoked slow hyperpolarization. For $\mathrm{vSWO}_{5-\mathrm{HT} 1 \mathrm{~B}}$ activation, brain slices were incubated with the external solution supplemented with $25 \mu \mathrm{M}$ of 9-cis-retinal, $0.025 \%( \pm)$ - $\alpha$-tocopherol, $0.2 \%$ essentially fatty acid-free albumin from bovine serum, and $0.1 \%$ dimethyl sulfoxide (Sigma). A train of $10473-\mathrm{nm}$ light flashes $(2.5-5.0 \mathrm{~mW}, 10 \mathrm{~Hz}$ ), each lasting $10 \mathrm{~ms}$, was delivered to induce $\mathrm{ChR2}$-assisted serotonin release. $\mathrm{vSWO}_{5-\mathrm{HT} 1 \mathrm{~B}}$ was activated by an illuminating of $380-\mathrm{nm}$ light $(40 \mu \mathrm{W})$ for $16 \mathrm{~s}$.

Measurement of Serotonin Release in Slices. Coronal slices $(250 \mu \mathrm{m}$ thick) containing the amygdala or DRN were prepared under red light, as described in the previous section, from Sert-Cre;orexin-ataxin3 mice injected with AAV-EF1 $\alpha$-DIO-SSFO-EYFP into the DRN. After 1-h incubation at room temperature in ACSF oxygenated with $\mathrm{O}_{2} 95 \%$ and $\mathrm{CO}_{2} 5 \%$, the slices were transferred to a microtube containing $120 \mu \mathrm{L}$ of ACSF. After 10-min incubation at $30^{\circ} \mathrm{C}, 70 \mu \mathrm{L}$ of the supernatant was collected for the measurement. After a brief and gentle wash by adding and discarding $70 \mu \mathrm{L}$ of ACSF to the slices, another $70 \mu \mathrm{L}$ of oxygenated ACSF was added for subsequent

1. Nishino S, Ripley B, Overeem S, Lammers GJ, Mignot E (2000) Hypocretin (orexin) deficiency in human narcolepsy. Lancet 355:39-40.

2. Peyron C, et al. (2000) A mutation in a case of early onset narcolepsy and a generalized absence of hypocretin peptides in human narcoleptic brains. Nat Med 6: 991-997.

3. Thannickal TC, et al. (2000) Reduced number of hypocretin neurons in human narcolepsy. Neuron 27:469-474.

4. Dauvilliers Y, Siegel JM, Lopez R, Torontali ZA, Peever JH (2014) Cataplexy-Clinica aspects, pathophysiology and management strategy. Nat Rev Neurol 10:386-395.

5. Sakurai T (2007) The neural circuit of orexin (hypocretin): Maintaining sleep and wakefulness. Nat Rev Neurosci 8:171-181.

6. Chemelli RM, et al. (1999) Narcolepsy in orexin knockout mice: Molecular genetics of sleep regulation. Cell 98:437-451.

7. Hara J, et al. (2001) Genetic ablation of orexin neurons in mice results in narcolepsy, hypophagia, and obesity. Neuron 30:345-354.

8. Hasegawa E, Yanagisawa M, Sakurai T, Mieda M (2014) Orexin neurons suppress narcolepsy via 2 distinct efferent pathways. J Clin Invest 124:604-616.

9. Scammell TE, Willie JT, Guilleminault C, Siegel JM; International Working Group on Rodent Models of Narcolepsy (2009) A consensus definition of cataplexy in mouse models of narcolepsy. Sleep 32:111-116.

10. Monti JM (2010) The structure of the dorsal raphe nucleus and its relevance to the regulation of sleep and wakefulness. Sleep Med Rev 14:307-317.

11. Imai $H$, Steindler DA, Kitai ST (1986) The organization of divergent axonal projections from the midbrain raphe nuclei in the rat. J Comp Neurol 243:363-380.

12. Steinbusch HW (1981) Distribution of serotonin-immunoreactivity in the central nervous system of the rat-cell bodies and terminals. Neuroscience 6:557-618.

13. McGinty DJ, Harper RM (1976) Dorsal raphe neurons: Depression of firing during sleep in cats. Brain Res 101:569-575.

14. Monti JM (2011) Serotonin control of sleep-wake behavior. Sleep Med Rev 15: 269-281.

15. Dayan P, Huys QJ (2009) Serotonin in affective control. Annu Rev Neurosci 32:95-126.

16. Liu Z, et al. (2014) Dorsal raphe neurons signal reward through 5-HT and glutamate. Neuron 81:1360-1374.

17. Miyazaki KW, et al. (2014) Optogenetic activation of dorsal raphe serotonin neurons enhances patience for future rewards. Curr Biol 24:2033-2040.

18. Warden MR, et al. (2012) A prefrontal cortex-brainstem neuronal projection that controls response to behavioural challenge. Nature 492:428-432.

19. Oishi Y, et al. (2013) Role of the medial prefrontal cortex in cataplexy. J Neurosci 33: 9743-9751.

20. Yizhar O, Fenno LE, Davidson TJ, Mogri M, Deisseroth K (2011) Optogenetics in neural systems. Neuron 71:9-34.

21. Yizhar O, et al. (2011) Neocortical excitation/inhibition balance in information processing and social dysfunction. Nature 477:171-178. sampling after $10 \mathrm{~min}$ of incubation. Samples were collected three times for baseline, and the slices were then illuminated with blue-light pulses $(20 \mathrm{~mW}$ $10 \mathrm{~ms}, 5 \mathrm{~Hz}$ for $1 \mathrm{~s}$ ). The supernatant was collected three times. Slices were illuminated once again and sampled three more times. After all sampling the serotonin concentration of each sample was measured via HPLC with a reverse-phase column (PP-ODS, Eicom) and an electrochemical detector (ECD-300, Eicom) to determine the extracellular levels of serotonin. The mobile phase consisted of $2.1 \mathrm{mM}$ sodium 1-decansulfonate, $0.1 \mathrm{mM}$ EDTA-

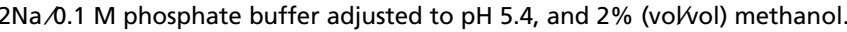
To eliminate data from the mice with unsuccessful AAV injections, mice whose DRN slices did not increase serotonin release by the light illumination were eliminated in the analyses for the amygdala (two of seven mice). Such a criterion was used because illumination of light to observe the fluorescence of SSFO-EYFP for checking SSFO expression before sampling would result in undesired activation of SSFO.

Statistics. All results are expressed as the mean \pm SEM. Comparisons between individuals were analyzed by two-tailed Student's $t$ test, whereas those within the individuals were analyzed by two-tailed Student's paired $t$ test. Welch's $t$ test was used when the variances of two group means were different. For the slice electrophysiological study, Friedman's test was performed. For the measurement of serotonin release, two-way repeated-measures ANOVA followed by Mann-Whitney's $U$ test, was performed.

ACKNOWLEDGMENTS. We thank X. Zhuang for Sert-Cre mice, K. Deisseroth for pAAV-DIO-SSFO-EYFP-WPRE-pA and pAAV-hSynl-EGFP-WPRE-pA, B. Roth for pAAV-DIO-hM4Di-mCherry and pAAV-DIO-hM3Dq-mCherry, R. Sprengel for iCre cDNA, and the Penn Vector Core for $p A A V 2-r h 10$. This study was supported in part by Grants-in-Aid for Scientific Research (B) (24390052, 16H05120) and for Challenging Exploratory Research (23659134) from the Japan Society for the Promotion of Science (JSPS) (to M.M.); a research grant of Astellas Foundation for Research on Metabolic Disorders and a research grant of the Brain Science Foundation (to M.M.); a JSPS Research Fellowship for Young Scientists (to E.H.); and by Deutsche Forschungsgemeinschaft Grants MA 4692/3-1 (to O.A.M.), He2471/18-1 Priority Program (SPP1926) and SFB874 project B10 (to S.H.)

22. Zhuang X, Masson J, Gingrich JA, Rayport S, Hen R (2005) Targeted gene expression in dopamine and serotonin neurons of the mouse brain. J Neurosci Methods 143:27-32.

23. Vassalli $A$, et al. (2013) Electroencephalogram paroxysmal $\theta$ characterizes cataplexy in mice and children. Brain 136:1592-1608.

24. Armbruster BN, Li X, Pausch MH, Herlitze S, Roth BL (2007) Evolving the lock to fit the key to create a family of $\mathrm{G}$ protein-coupled receptors potently activated by an inert ligand. Proc Natl Acad Sci USA 104:5163-5168.

25. Boissard R, et al. (2002) The rat ponto-medullary network responsible for paradoxical sleep onset and maintenance: A combined microinjection and functional neuroanatomical study. Eur J Neurosci 16:1959-1973.

26. Fuller PM, Saper CB, Lu J (2007) The pontine REM switch: Past and present. J Physiol 584:735-741.

27. Lu J, Sherman D, Devor M, Saper CB (2006) A putative flip-flop switch for control of REM sleep. Nature 441:589-594.

28. Luppi $\mathrm{PH}$ et al. (2011) The neuronal network responsible for paradoxical sleep and its dysfunctions causing narcolepsy and rapid eye movement (REM) behavior disorder. Sleep Med Rev 15:153-163.

29. Van Dort CJ, et al. (2015) Optogenetic activation of cholinergic neurons in the PPT or LDT induces REM sleep. Proc Natl Acad Sci USA 112:584-589.

30. Honda K, Riehl J, Mignot E, Nishino S (1999) Dopamine D3 agonists into the substantia nigra aggravate cataplexy but do not modify sleep. Neuroreport 10 3717-3724.

31. Okura M, Riehl J, Mignot E, Nishino S (2000) Sulpiride, a D2/D3 blocker, reduces cataplexy but not REM sleep in canine narcolepsy. Neuropsychopharmacol 23:528-538.

32. Janak PH, Tye KM (2015) From circuits to behaviour in the amygdala. Nature 517 284-292.

33. Burgess CR, Oishi Y, Mochizuki T, Peever JH, Scammell TE (2013) Amygdala lesions reduce cataplexy in orexin knock-out mice. J Neurosci 33:9734-9742.

34. Shimshek DR, et al. (2002) Codon-improved Cre recombinase (iCre) expression in the mouse. Genesis 32:19-26.

35. Marcus JN, et al. (2001) Differential expression of orexin receptors 1 and 2 in the rat brain. J Comp Neurol 435:6-25.

36. Peyron C, et al. (1998) Neurons containing hypocretin (orexin) project to multiple neuronal systems. J Neurosci 18:9996-10015.

37. Liu M, Blanco-Centurion C, Konadhode RR, Luan L, Shiromani PJ (2016) Orexin gene transfer into the amygdala suppresses both spontaneous and emotion-induced cataplexy in orexin-knockout mice. Eur J Neurosci 43:681-688.

38. Sears RM, et al. (2013) Orexin/hypocretin system modulates amygdala-dependent threat learning through the locus coeruleus. Proc Natl Acad Sci USA 110:20260-20265.

39. Soya $S$, et al. (2013) Orexin receptor-1 in the locus coeruleus plays an important role in cue-dependent fear memory consolidation. J Neurosci 33:14549-14557.

40. Masseck OA, et al. (2014) Vertebrate cone opsins enable sustained and highly sensitive rapid control of Gi/o signaling in anxiety circuitry. Neuron 81:1263-1273. 
41. Clark EL, Baumann CR, Cano G, Scammell TE, Mochizuki T (2009) Feeding-elicited cataplexy in orexin knockout mice. Neuroscience 161:970-977.

42. España RA, McCormack SL, Mochizuki T, Scammell TE (2007) Running promotes wakefulness and increases cataplexy in orexin knockout mice. Sleep 30:1417-1425.

43. Nishino S, Mignot E (1997) Pharmacological aspects of human and canine narcolepsy. Prog Neurobiol 52:27-78.

44. Gulyani S, Wu MF, Nienhuis R, John J, Siegel JM (2002) Cataplexy-related neurons in the amygdala of the narcoleptic dog. Neuroscience 112:355-365.

45. Hong SB, Tae WS, Joo EY (2006) Cerebral perfusion changes during cataplexy in narcolepsy patients. Neurology 66:1747-1749.

46. Meletti S, et al. (2015) The brain correlates of laugh and cataplexy in childhood narcolepsy. J Neurosci 35:11583-11594.

47. Jha SK, Ross RJ, Morrison AR (2005) Sleep-related neurons in the central nucleus of the amygdala of rats and their modulation by the dorsal raphe nucleus. Physiol Behav 86:415-426.

48. Oka T, Tsumori T, Yokota S, Yasui Y (2008) Neuroanatomical and neurochemical organization of projections from the central amygdaloid nucleus to the nucleus retroambiguus via the periaqueductal gray in the rat. Neurosci Res 62:286-298.

49. Rizvi TA, Ennis M, Behbehani MM, Shipley MT (1991) Connections between the central nucleus of the amygdala and the midbrain periaqueductal gray: topography and reciprocity. $J$ Comp Neurol 303:121-131.

50. Boissard R, Fort P, Gervasoni D, Barbagli B, Luppi PH (2003) Localization of the GABAergic and non-GABAergic neurons projecting to the sublaterodorsal nucleus and potentially gating paradoxical sleep onset. Eur J Neurosci 18:1627-1639.

51. Fung SJ, et al. (2011) Projection neurons from the central nucleus of the amygdala to the nucleus pontis oralis. J Neurosci Res 89:429-436.

52. Xi M, Fung SJ, Sampogna S, Chase MH (2011) Excitatory projections from the amygdala to neurons in the nucleus pontis oralis in the rat: an intracellular study. Neuroscience 197:181-190.

53. Rainnie DG (1999) Serotonergic modulation of neurotransmission in the rat basolateral amygdala. J Neurophysio/ 82:69-85.

54. Stutzmann GE, LeDoux JE (1999) GABAergic antagonists block the inhibitory effects of serotonin in the lateral amygdala: A mechanism for modulation of sensory inputs related to fear conditioning. J Neurosci 19:RC8.

55. Bocchio $M$, et al. (2015) Increased serotonin transporter expression reduces fear and recruitment of parvalbumin interneurons of the amygdala. Neuropsychopharmacology 40:3015-3026.

56. Ponz A, et al. (2010) Abnormal activity in reward brain circuits in human narcolepsy with cataplexy. Ann Neurol 67:190-200.

57. Ponz A, et al. (2010) Reduced amygdala activity during aversive conditioning in human narcolepsy. Ann Neurol 67:394-398.

58. Schwartz S, et al. (2008) Abnormal activity in hypothalamus and amygdala during humour processing in human narcolepsy with cataplexy. Brain 131:514-522.

59. Bocchio M, McHugh SB, Bannerman DM, Sharp T, Capogna M (2016) Serotonin, amygdala and fear: Assembling the puzzle. Front Neural Circuits 10:24.

60. Burghardt NS, Bush DE, McEwen BS, LeDoux JE (2007) Acute selective serotonin reuptake inhibitors increase conditioned fear expression: Blockade with a 5-HT(2C) receptor antagonist. Biol Psychiatry 62:1111-1118.

61. Izumi T, Inoue T, Kitaichi Y, Nakagawa S, Koyama T (2006) Target brain sites of the anxiolytic effect of citalopram, a selective serotonin reuptake inhibitor. Eur $J$ Pharmacol 534:129-132.
62. Johnson PL, et al. (2015) Pharmacological depletion of serotonin in the basolateral amygdala complex reduces anxiety and disrupts fear conditioning. Pharmacol Biochem Behav 138:174-179.

63. Kitaichi $Y$, et al. (2014) Local infusion of citalopram into the basolateral amygdala decreased conditioned fear of rats through increasing extracellular serotonin levels. Prog Neuropsychopharmacol Biol Psychiatry 54:216-222.

64. Inoue T, et al. (2004) Selective serotonin reuptake inhibitor reduces conditioned fear through its effect in the amygdala. Eur J Pharmacol 497:311-316.

65. Izumi T, et al. (2012) Effects of serotonergic terminal lesion in the amygdala on conditioned fear and innate fear in rats. Eur J Pharmacol 696:89-95.

66. Stuber GD, et al. (2011) Excitatory transmission from the amygdala to nucleus accumbens facilitates reward seeking. Nature 475:377-380.

67. Garavan H, Pendergrass JC, Ross TJ, Stein EA, Risinger RC (2001) Amygdala response to both positively and negatively valenced stimuli. Neuroreport 12:2779-2783.

68. Everitt BJ, Cador M, Robbins TW (1989) Interactions between the amygdala and ventral striatum in stimulus-reward associations: Studies using a second-order schedule of sexual reinforcement. Neuroscience 30:63-75.

69. Hatfield T, Han JS, Conley M, Gallagher M, Holland P (1996) Neurotoxic lesions of basolateral, but not central, amygdala interfere with Pavlovian second-order conditioning and reinforcer devaluation effects. J Neurosci 16:5256-5265.

70. Nishijo $H$, Ono $T$, Nishino $H$ (1988) Single neuron responses in amygdala of alert monkey during complex sensory stimulation with affective significance. $J$ Neurosci 8:3570-3583.

71. Paton JJ, Belova MA, Morrison SE, Salzman CD (2006) The primate amygdala represents the positive and negative value of visual stimuli during learning. Nature 439: 865-870.

72. Schoenbaum G, Chiba AA, Gallagher M (1998) Orbitofrontal cortex and basolateral amygdala encode expected outcomes during learning. Nat Neurosci 1:155-159.

73. Tye KM, Stuber GD, de Ridder B, Bonci A, Janak PH (2008) Rapid strengthening of thalamo-amygdala synapses mediates cue-reward learning. Nature 453:1253-1257.

74. Gore F, et al. (2015) Neural representations of unconditioned stimuli in basolateral amygdala mediate innate and learned responses. Cell 162:134-145.

75. Namburi P, et al. (2015) A circuit mechanism for differentiating positive and negative associations. Nature 520:675-678.

76. McCool BA, Christian DT, Fetzer JA, Chappell AM (2014) Lateral/basolateral amygdala serotonin type-2 receptors modulate operant self-administration of a sweetened ethanol solution via inhibition of principal neuron activity. Front Integr Nuerosci 8:5.

77. Borgland SL, Taha SA, Sarti F, Fields HL, Bonci A (2006) Orexin A in the VTA is critical for the induction of synaptic plasticity and behavioral sensitization to cocaine. Neuron 49:589-601.

78. Harris GC, Wimmer M, Aston-Jones G (2005) A role for lateral hypothalamic orexin neurons in reward seeking. Nature 437:556-559.

79. Narita M, et al. (2006) Direct involvement of orexinergic systems in the activation of the mesolimbic dopamine pathway and related behaviors induced by morphine. J Neurosci 26:398-405.

80. Tabuchi S, et al. (2014) Conditional ablation of orexin/hypocretin neurons: A new mouse model for the study of narcolepsy and orexin system function. J Neurosci 34: 6495-6509.

81. Mieda M, et al. (2015) Cellular clocks in AVP neurons of the SCN are critical for interneuronal coupling regulating circadian behavior rhythm. Neuron 85:1103-1116.

82. Paxinos G, Franklin KBJ (2001) The Mouse Brain in Stereotaxic Coordinates, 2nd Ed. (Academic, San Diego). 


\section{Supporting Information}

\section{Hasegawa et al. 10.1073/pnas.1614552114}

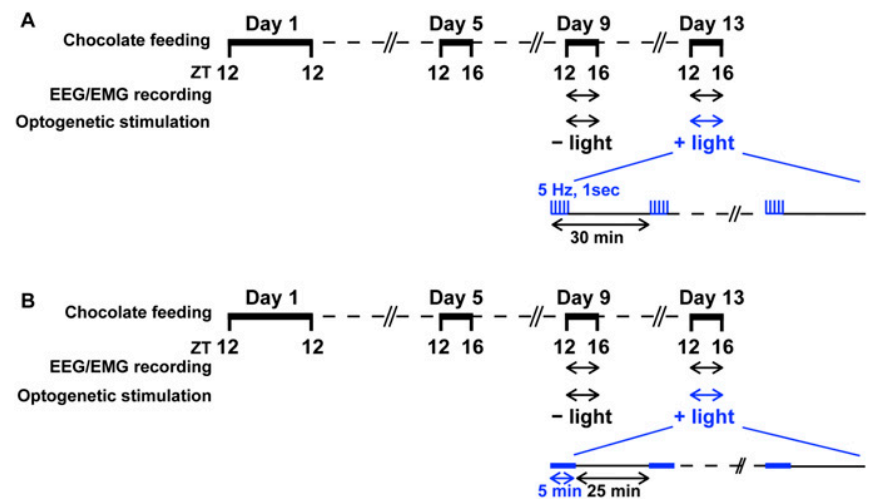

Fig. S1. Scheme of chocolate feeding, EEG/EMG recording, and photostimulation protocol. (A) Optogenetic activation of neurons and nerve fibers expressing SSFO. (B) Optogenetic inhibition of nerve fibers expressing vSWO ${ }_{5-\mathrm{HT} 1 \mathrm{~B}}$. Thick horizontal bars represent times of chocolate feeding. On day 9 , EEG/EMG was recorded during $4 \mathrm{~h}$ of chocolate feeding without photostimulation. On day 13, EEG/EMG recording was repeated with photostimulation of 10 -ms pulses at $5 \mathrm{~Hz}$ for $1 \mathrm{~s}$ delivered every $30 \mathrm{~min}(A)$ or a 5-min pulse delivered every $30 \mathrm{~min}(B)$ during $4 \mathrm{~h}$ of chocolate feeding. ZT, zeitgeber time.
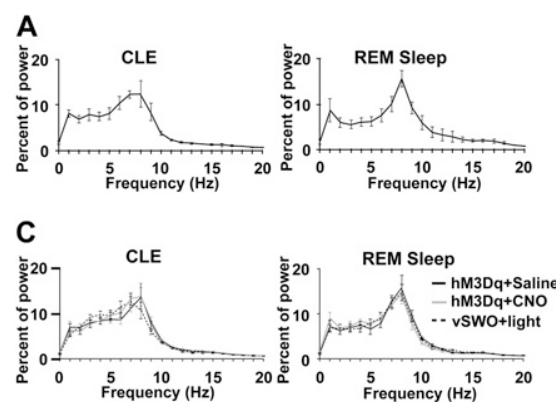

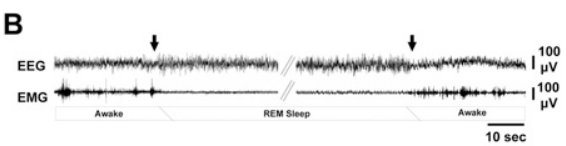

D

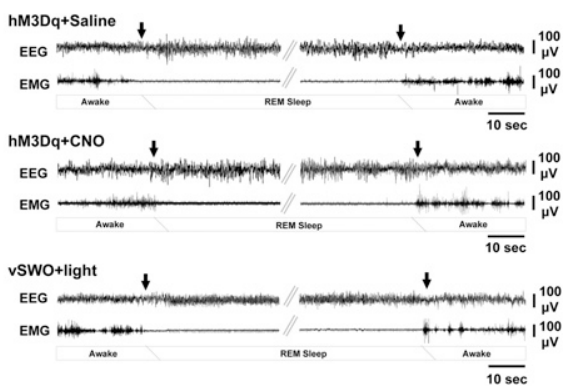

Fig. S2. Characteristics of EEG/EMG during CLEs. (A) Power spectral analysis of EEG by FFT during CLEs and REM sleep in unstimulated Sert-Cre;orexin-ataxin3 mice with SSFO expression in DRN serotonin neurons and an optic fiber above the DRN but without optogenetic stimulation ( $n=5$ ). Panels reflect mean spectra of five mice. Data are standardized by expressing each frequency bin as a percentage relative to the total power of the same epochs. (B) A typical EEG/EMG trace during a CLE as described in A. Arrows demarcate onsets and terminations of a CLE. (C) Power spectral analysis of EEG by FFT during CLEs and REM sleep in either orexin-ataxin3 mice expressing hM3Dq in the amygdala and administered saline or $\mathrm{CNO}_{\text {or }} \mathrm{Ox} 1 \mathrm{r}^{-/-} \mathrm{Ox} 2 \mathrm{r}^{-/-}$mice expressing $\mathrm{OX} 2 \mathrm{R}$ and $\mathrm{vSWO} \mathrm{O}_{5-\mathrm{HT} 1 \mathrm{~B}}$ in DRN serotonin neurons whose amygdalae were optogenetically stimulated $(n=4,4$, and 5 , respectively). Values are mean \pm SEM. ( $D$ ) Typical EEG/EMG traces during CLEs in mice described in $C$. 
A Amygdala

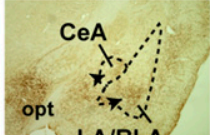

CeA

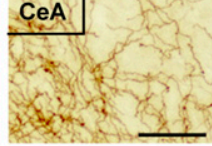

LA/BLA

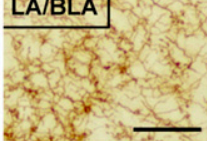

SNc
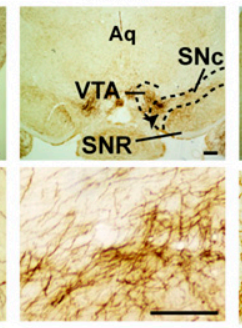

B

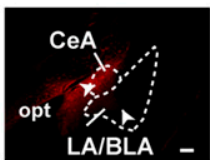

C

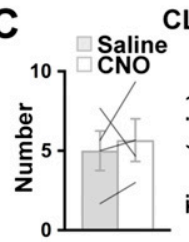

CLE

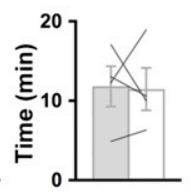

VIPAG
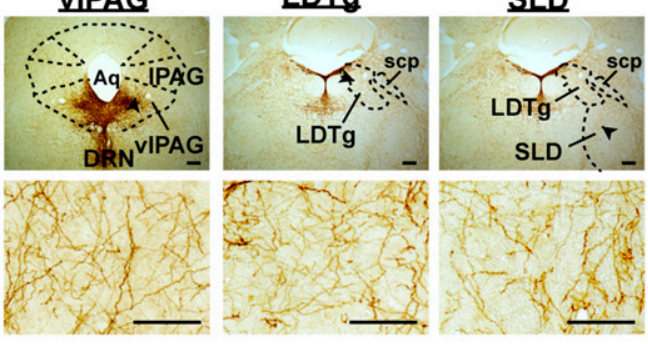

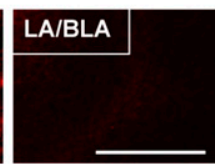

Wakefulness

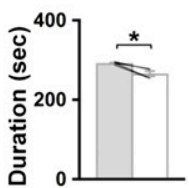

Fig. S3. Projections of DRN serotonin neurons and effects of chemogenetic inhibition of the CeA on CLEs. ( $A$ ) Coronal brain sections containing the DRN prepared from Sert-Cre;orexin-ataxin3 mice with targeted injection of AAV-EF1 $\alpha$-DIO-SSFO-EYFP in the DRN were stained brown with anti-GFP antibody. Particularly dense staining of EYFP ${ }^{+}$fibers was observed in the amygdala, as well as in the SNc. Other REM-regulating nuclei (e.g., vIPAG, LDTg, and SLD) also receive DRN serotonin projections. Regions denoted by black arrowheads are shown at higher magnification. (Scale bars, $100 \mu \mathrm{m}$.) $(B$ and $C$ ) $C$ hemogenetic inhibition of the CeA does not suppress CLEs in orexin-ataxin3 mice. $(B)$ Coronal brain sections of the amygdala prepared from orexin-ataxin3 mice with a bilateral injection of AAV-Synl-iCre and AAV-EF1 $\alpha$-DIO-hM4Di-mCherry in the amygdala were stained with anti-mCherry (red) antibody. Unlike in mice described in Fig. 5A, hM4Di-mCherry was expressed in the CeA but not in the BLA in several mice, because of slight differences in AAV injection sites. Regions denoted by white arrowheads are shown at higher magnification. (Scale bars, $100 \mu \mathrm{m}$.) (C) Orexin-ataxin3 mice with hM4Di-mCherry expression in the CeA were injected with saline or CNO at the onset of the dark phase. The episode number of and time spent in CLEs, and mean duration of wakefulness episodes during the 12-h dark phase without chocolate feeding are shown $(n=4)$. The mice represent a subset different from that with hM4Di expression in both the $\mathrm{CeA}$ and BLA shown in Fig. 5. Bar graphs show mean $\pm \mathrm{SEM},{ }^{*} P<0.05$, two-tailed Student's paired $t$ test. 
A Amygdala SSFO(-) +light
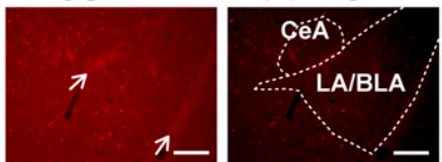

Amygdala SSFO(+) +light

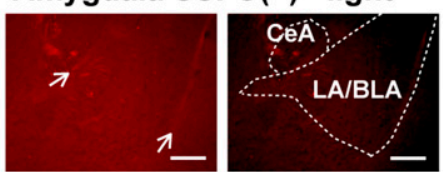

B Amygdala hM4Di
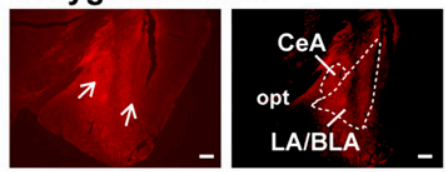

CeA hM4Di
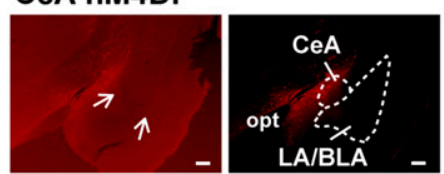

Amygdala hM3Dq
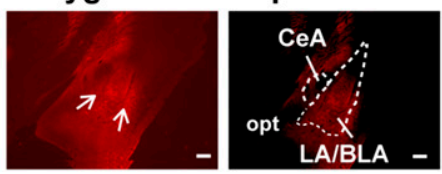

\section{Amygdala vSWO}

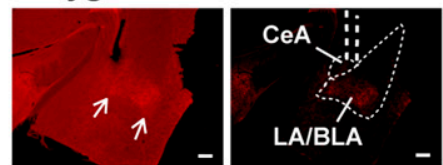

D Amygdala hM3Dq Amygdala hM4Di

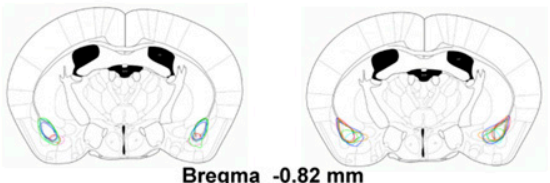

Bregma $-0.82 \mathrm{~mm}$


Fig. S4. Anatomy of immunostained amygdala sections and expression of hM3Dq or hM4Di in orexin-ataxin3 mice receiving an AAV injection in the amygdala. ( $A-C$ ) Amygdala sections shown in Figs. $4 B, 5 A$ and $C$, and $6 B$ and Fig. S3 (Right) are overexposed to show anatomical landmarks (Left). White arrows indicate the external and intermediate capsules. (Scale bars, $100 \mu \mathrm{m}$.) $(D)$ Schematics show the spread of hM3Dq-mCherry or hM4Di-mCherry expression. Different colored lines represent the four (hM3Dq) or seven (hM4Di) different mice of the orexin-ataxin3 group with an injection of AAV-Synl-iCre and AAV-EF1 $\alpha$-DIO-hM3Dq$m$ Cherry or AAV-EF1 $\alpha$-DIO-hM4Di-mCherry. Drawings are adapted from a mouse brain atlas (82), and anteroposterior coordinates are relative to bregma.

\section{A Amygdala OX1R;0X2R}
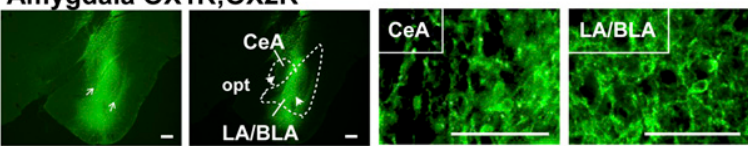

B
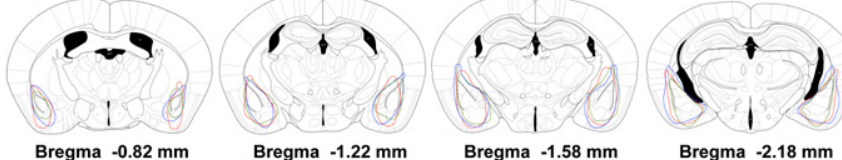

Fig. S5. Expression of orexin receptors in the amygdala of $O \times 1 r^{-1-} O \times 2 r^{-1-}$ mice with restored orexin receptors in the amygdala. (A) Coronal brain sections of the amygdala prepared from $O \times 1 r^{-1-} O \times 2 r^{-1-}$ mice that bilaterally received an AAV microinjection (a mixture of recombinant AAV vectors AAV-EF1a-Ox1r-EGFP and AAV-EF1a-Ox2r-EGFP) were stained with anti-GFP (green) antibody. Regions denoted by white arrowheads are shown at higher magnification. The leftmost image is an overexposed section of the second-leftmost image to show anatomical landmarks. (Scale bars, $100 \mu \mathrm{m}$.) $(B) \mathrm{Schematics} \mathrm{show} \mathrm{the} \mathrm{spread} \mathrm{of}$ orexin receptor expression. Different colored lines represent the three different mice. Drawings are adapted from a mouse brain atlas (82), and anteroposterior coordinates are relative to bregma. 


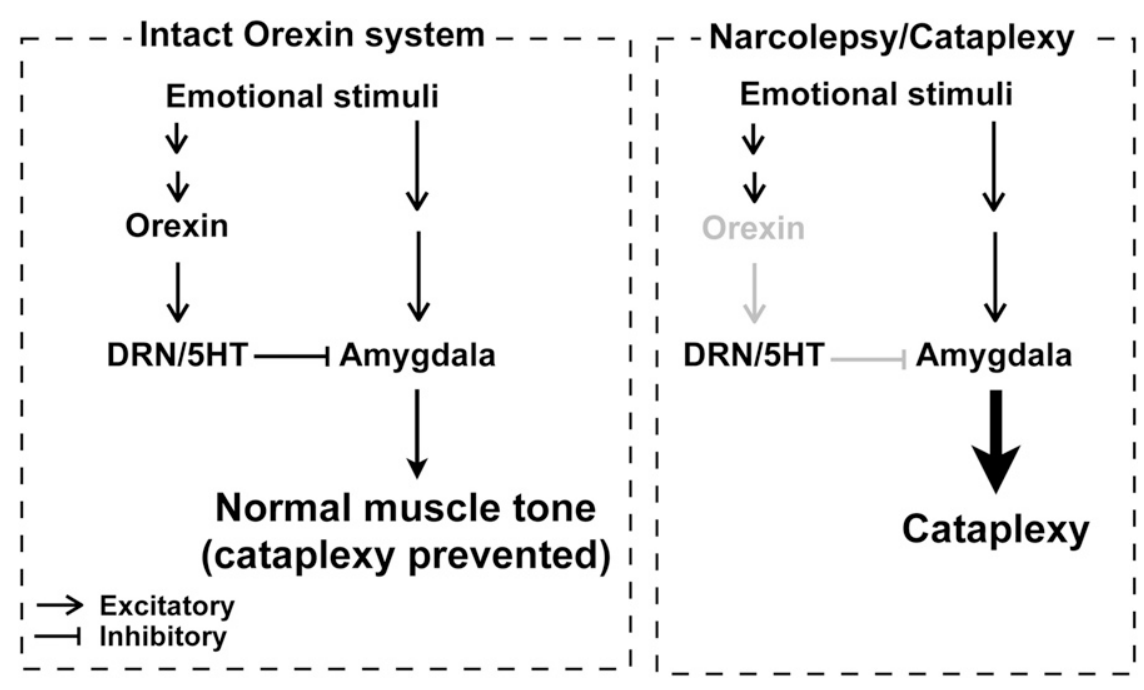

Fig. S6. Model of the neural pathway by which orexin neurons prevent cataplexy. In the absence of orexin neurons (type 1 narcolepsy), the amygdala overresponds to emotional stimuli and sends exaggerated output to induce cataplexy. Orexin neurons activate DRN serotonin neurons, which reduce amygdala activity and prevent cataplexy.

Table S1. Vigilance state parameters recorded from Sert-Cre;orexin-ataxin3 mice receiving photostimulation in the DRN

\begin{tabular}{|c|c|c|c|c|}
\hline \multirow[b]{2}{*}{ Parameter } & \multicolumn{2}{|c|}{ DRN SSFO(+) $(n=5)$} & \multicolumn{2}{|c|}{ DRN SSFO(-) $(n=5)$} \\
\hline & -Light & +Light & -Light & +Light \\
\hline \multicolumn{5}{|l|}{ Wakefulness } \\
\hline Total time (min) & $164.2 \pm 6.0$ & $176.9 \pm 6.3$ & $162.6 \pm 7.8$ & $148.3 \pm 8.0$ \\
\hline Episode duration (s) & $320.2 \pm 20.7$ & $368.4 \pm 28.0$ & $310.8 \pm 31.3$ & $286.0 \pm 21.2$ \\
\hline Episode no. & $26.0 \pm 2.9$ & $23.0 \pm 0.9$ & $28.8 \pm 1.4$ & $25.6 \pm 1.4$ \\
\hline \multicolumn{5}{|l|}{ NREM sleep } \\
\hline Total time (min) & $48.1 \pm 6.6$ & $50.4 \pm 6.6$ & $49.2 \pm 5.1$ & $64.6 \pm 8.3$ \\
\hline Episode duration (s) & $124.0 \pm 14.2$ & $129.6 \pm 15.4$ & $131.8 \pm 17.3$ & $198.0 \pm 24.3^{*}$ \\
\hline Episode no. & $21.4 \pm 2.8$ & $22.6 \pm 1.4$ & $22.0 \pm 1.6$ & $19.4 \pm 1.4$ \\
\hline \multicolumn{5}{|l|}{ REM sleep } \\
\hline Total time (min) & $13.9 \pm 2.0$ & $10.9 \pm 1.5^{*}$ & $12.4 \pm 2.6$ & $14.5 \pm 1.2$ \\
\hline Episode duration (s) & $87.1 \pm 8.2$ & $96.0 \pm 8.5$ & $80.9 \pm 3.8$ & $70.9 \pm 4.9$ \\
\hline Episode no. & $8.0 \pm 0.9$ & $7.0 \pm 1.4$ & $9.0 \pm 1.8$ & $7.8 \pm 1.1$ \\
\hline Latency (s) & $120.0 \pm 13.2$ & $159.7 \pm 23.4$ & $147.8 \pm 27.9$ & $223.7 \pm 30.0$ \\
\hline \multicolumn{5}{|l|}{ CLE } \\
\hline Total time $(\mathrm{min})$ & $13.8 \pm 1.2$ & $1.3 \pm 1.1 * * *$ & $15.9 \pm 1.3$ & $12.5 \pm 1.4$ \\
\hline Episode duration (s) & $131.8 \pm 11.2$ & $64.5 \pm 18.2^{*}$ & $135.0 \pm 11.7$ & $110.4 \pm 10.8$ \\
\hline Episode no. & $6.4 \pm 0.5$ & $1.2 \pm 0.5^{* *}$ & $7.2 \pm 0.7$ & $6.8 \pm 0.4$ \\
\hline
\end{tabular}

SSFO(-) represents mice without SSFO expression used to gauge the effect of light elimination. EEG/EMG during $4 \mathrm{~h}$ of chocolate feeding were analyzed. Values are mean $\pm \mathrm{SEM}, * P<0.05, * * P<0.01, * * * P<0.001$, twotailed Student's paired $t$ test. 
Table S2. Vigilance state parameters recorded from Sert-Cre;orexin-ataxin3 mice receiving photostimulation at serotonin fibers in the amygdala or SNc

\begin{tabular}{|c|c|c|c|c|c|c|}
\hline \multirow[b]{2}{*}{ Parameter } & \multicolumn{2}{|c|}{ Amygdala SSFO(+) $(n=6)$} & \multicolumn{2}{|c|}{ Amygdala SSFO(-) $(n=4)$} & \multicolumn{2}{|c|}{$\operatorname{SNc} \operatorname{SSFO}(+)(n=4)$} \\
\hline & -Light & + Light & -Light & + Light & -Light & + Light \\
\hline \multicolumn{7}{|l|}{ Wakefulness } \\
\hline Total time (min) & $173.2 \pm 7.9$ & $156.6 \pm 13.7$ & $179.6 \pm 6.1$ & $154.7 \pm 2.3^{*}$ & $190.9 \pm 3.5$ & $179.2 \pm 10.7$ \\
\hline Episode duration (s) & $333.5 \pm 20.4$ & $355.7 \pm 70.2$ & $320.8 \pm 33.7$ & $317.3 \pm 22.5$ & $345.0 \pm 17.6$ & $351.5 \pm 29.7$ \\
\hline Episode no. & $27.8 \pm 1.4$ & $24.8 \pm 0.9$ & $30.8 \pm 2.1$ & $24.5 \pm 1.8$ & $27.5 \pm 1.9$ & $25.5 \pm 1.3$ \\
\hline \multicolumn{7}{|l|}{ NREM sleep } \\
\hline Total time $(\mathrm{min})$ & $46.6 \pm 6.3$ & $73.7 \pm 12.6^{*}$ & $41.7 \pm 4.6$ & $63.2 \pm 1.9 * *$ & $27.6 \pm 1.9$ & $39.5 \pm 8.1$ \\
\hline Episode duration (s) & $125.7 \pm 16.4$ & $185.0 \pm 19.1^{*}$ & $99.5 \pm 8.9$ & $189.5 \pm 8.9 * * *$ & $80.8 \pm 12.8$ & $117.5 \pm 23.8$ \\
\hline Episode no. & $14.2 \pm 0.9$ & $7.8 \pm 0.9$ & $25.3 \pm 2.1$ & $19.5 \pm 1.0$ & $15.3 \pm 1.5$ & $13.5 \pm 1.2$ \\
\hline \multicolumn{7}{|l|}{ REM sleep } \\
\hline Total time (min) & $9.3 \pm 1.6$ & $9.1 \pm 1.9$ & $7.2 \pm 2.1$ & $10.5 \pm 0.9$ & $8.6 \pm 1.0$ & $8.6 \pm 3.0$ \\
\hline Episode duration (s) & $69.3 \pm 5.7$ & $85.1 \pm 5.9$ & $91.2 \pm 7.4$ & $89.7 \pm 10.2$ & $67.5 \pm 4.8$ & $64.6 \pm 15.3$ \\
\hline Episode no. & $7.7 \pm 1.1$ & $7.5 \pm 0.8$ & $5.0 \pm 1.6$ & $7.3 \pm 0.5$ & $7.8 \pm 0.9$ & $6.8 \pm 1.9$ \\
\hline Latency (s) & $141.6 \pm 21.8$ & $184.7 \pm 16.9$ & $146.3 \pm 14.6$ & $170.0 \pm 10.3$ & $100.6 \pm 18.0$ & $108.4 \pm 35.5$ \\
\hline \multicolumn{7}{|l|}{ CLE } \\
\hline Total time (min) & $11.0 \pm 0.8$ & $0.5 \pm 0.3 * * *$ & $11.6 \pm 0.8$ & $11.4 \pm 2.3$ & $12.9 \pm 1.3$ & $12.7 \pm 0.9$ \\
\hline Episode duration (s) & $104.3 \pm 11.0$ & $31.3 \pm 20.1$ & $103.6 \pm 8.5$ & $101.3 \pm 19.3$ & $103.0 \pm 6.7$ & $114.6 \pm 15.3$ \\
\hline Episode no. & $6.5 \pm 0.5$ & $0.3 \pm 0.2^{* * *}$ & $6.8 \pm 0.5$ & $6.8 \pm 0.3$ & $7.5 \pm 0.6$ & $6.8 \pm 0.8$ \\
\hline
\end{tabular}

SSFO(-) represents mice without SSFO expression used to gauge the effect of light elimination. EEG/EMG during $4 \mathrm{~h}$ of chocolate feeding was analyzed. Values are mean $\pm \mathrm{SEM},{ }^{*} P<0.05, * * P<0.01, * * * P<0.001$, two-tailed Student's paired $t$ test.

Table S3. Vigilance state parameters recorded from Sert-Cre;orexin-ataxin3 mice receiving photostimulation at serotonin fibers in the vIPAG, LDTg, or SLD

\begin{tabular}{|c|c|c|c|c|c|c|}
\hline \multirow[b]{2}{*}{ Parameter } & \multicolumn{2}{|c|}{$\operatorname{vIPAG~SSFO}(+)(n=5)$} & \multicolumn{2}{|c|}{ LDTg SSFO(+) $(n=4)$} & \multicolumn{2}{|c|}{$\operatorname{SLD~SSFO}(+)(n=4)$} \\
\hline & -Light & + Light & -Light & + Light & -Light & + Light \\
\hline \multicolumn{7}{|l|}{ Wakefulness } \\
\hline Total time $(\min )$ & $168.8 \pm 10.6$ & $165.7 \pm 7.9$ & $142.5 \pm 14.5$ & $133.3 \pm 16.4$ & $150.3 \pm 8.9$ & $134.7 \pm 8.2 *$ \\
\hline Episode duration (s) & $328.4 \pm 27.0$ & $326.4 \pm 18.4$ & $289.3 \pm 18.0$ & $269.0 \pm 17.0$ & $307.5 \pm 20.8$ & $285.5 \pm 21.8$ \\
\hline Episode no. & $27.2 \pm 1.5$ & $26.0 \pm 0.9$ & $27.3 \pm 2.5$ & $24.5 \pm 1.7$ & $26.8 \pm 1.8$ & $27.3 \pm 1.3$ \\
\hline \multicolumn{7}{|l|}{ NREM sleep } \\
\hline Total time (min) & $47.0 \pm 7.8$ & $54.7 \pm 6.6$ & $67.7 \pm 12.2$ & $74.4 \pm 16.0$ & $63.6 \pm 10.4$ & $79.5 \pm 9.0 * *$ \\
\hline Episode duration (s) & $127.6 \pm 16.2$ & $169.8 \pm 20.2$ & $208.3 \pm 50.9$ & $230.8 \pm 51.0$ & $184.3 \pm 30.1$ & $220.3 \pm 26.5$ \\
\hline Episode no. & $21.4 \pm 1.4$ & $19.2 \pm 0.9$ & $20.8 \pm 2.3$ & $19.3 \pm 1.3$ & $20.3 \pm 0.5$ & $21.0 \pm 0.7$ \\
\hline \multicolumn{7}{|l|}{ REM sleep } \\
\hline Total time (min) & $9.9 \pm 2.4$ & $3.0 \pm 1.0$ & $13.6 \pm 3.6$ & $14.7 \pm 3.4$ & $15.2 \pm 1.0$ & $17.2 \pm 1.9$ \\
\hline Episode duration (s) & $82.9 \pm 12.2$ & $80.1 \pm 7.5$ & $84.8 \pm 7.6$ & $94.3 \pm 2.1$ & $121.6 \pm 4.4$ & $110.5 \pm 11.8$ \\
\hline Episode no. & $7.0 \pm 1.3$ & $3.0 \pm 1.0$ & $9.3 \pm 1.7$ & $9.3 \pm 1.9$ & $7.5 \pm 0.5$ & $9.5 \pm 1.0$ \\
\hline Latency (s) & $137.9 \pm 19.5$ & $167.8 \pm 14.7$ & $207.3 \pm 42.3$ & $209.6 \pm 40.0$ & $189.2 \pm 39.4$ & $189.8 \pm 13.7$ \\
\hline \multicolumn{7}{|l|}{ CLE } \\
\hline Total time (min) & $14.3 \pm 1.9$ & $13.7 \pm 1.1$ & $16.3 \pm 2.0$ & $17.6 \pm 4.0$ & $11.0 \pm 1.6$ & $8.6 \pm 1.3$ \\
\hline Episode duration (s) & $125.8 \pm 13.4$ & $107.8 \pm 6.4$ & $129.4 \pm 13.8$ & $161.4 \pm 41.0$ & $96.8 \pm 7.4$ & $77.1 \pm 11.8$ \\
\hline Episode no. & $6.8 \pm 0.5$ & $7.6 \pm 0.2$ & $7.5 \pm 0.3$ & $6.8 \pm 0.5$ & $6.8 \pm 0.5$ & $6.8 \pm 1.0$ \\
\hline
\end{tabular}

SSFO(-) represents mice without SSFO expression used to gauge the effect of light elimination. EEG/EMG during $4 \mathrm{~h}$ of chocolate feeding was analyzed. Values are mean $\pm \mathrm{SEM}, * P<0.05, * * P<0.01$, two-tailed Student's paired $t$ test. 
Table S4. Vigilance state parameters recorded from orexin-ataxin3 mice receiving hM4Di or hM3Dq stimulation in the amygdala

\begin{tabular}{|c|c|c|c|c|c|c|}
\hline \multirow[b]{2}{*}{ Parameter } & \multicolumn{2}{|c|}{ Amygdala hM4Di $(n=7)$} & \multicolumn{2}{|c|}{ CeA hM4Di $(n=4)$} & \multicolumn{2}{|c|}{ Amygdala hM3Dq $(n=6)$} \\
\hline & Saline & $\mathrm{CNO}$ & Saline & $\mathrm{CNO}$ & Saline & $\mathrm{CNO}$ \\
\hline \multicolumn{7}{|l|}{ 12-h dark phase } \\
\hline \multicolumn{7}{|l|}{ Wakefulness } \\
\hline Total time $(\mathrm{min})$ & $380.9 \pm 14.0$ & $338.1 \pm 11.7 * *$ & $384.9 \pm 7.5$ & $362.5 \pm 9.7$ & $408.1 \pm 19.4$ & $413.9 \pm 8.4$ \\
\hline Episode duration (s) & $287.1 \pm 7.0$ & $282.7 \pm 10.4$ & $292.2 \pm 3.5$ & $265.8 \pm 6.5^{*}$ & $324.7 \pm 20.1$ & $339.5 \pm 44.9$ \\
\hline Episode no. & $79.0 \pm 3.0$ & $70.0 \pm 3.4^{*}$ & $79.3 \pm 1.1$ & $80.4 \pm 2.5$ & $75.2 \pm 5.3$ & $74.9 \pm 9.1$ \\
\hline \multicolumn{7}{|l|}{ NREM sleep } \\
\hline Total time (min) & $281.9 \pm 15.0$ & $333.1 \pm 14.9 * *$ & $275.9 \pm 10.4$ & $301.9 \pm 11.4$ & $252.6 \pm 19.5$ & $241.5 \pm 10.1$ \\
\hline Episode duration (s) & $217.6 \pm 18.0$ & $268.9 \pm 21.2^{*}$ & $209.7 \pm 9.1$ & $221.6 \pm 11.1$ & $212.1 \pm 28.5$ & $217.5 \pm 20.6$ \\
\hline Episode no. & $77.4 \pm 3.0$ & $73.9 \pm 3.3$ & $78.3 \pm 1.0$ & $79.8 \pm 0.8$ & $71.4 \pm 5.4$ & $67.5 \pm 9.7$ \\
\hline \multicolumn{7}{|l|}{ REM sleep } \\
\hline Total time $(\min )$ & $46.3 \pm 2.2$ & $47.7 \pm 4.0$ & $47.4 \pm 1.0$ & $43.9 \pm 1.8$ & $49.2 \pm 1.9$ & $41.3 \pm 2.0$ \\
\hline Episode duration (s) & $99.6 \pm 3.9$ & $114.8 \pm 5.5^{* *}$ & $101.5 \pm 3.5$ & $104.6 \pm 6.8$ & $101.9 \pm 4.6$ & $110.6 \pm 5.8^{*}$ \\
\hline Episode no. & $28.0 \pm 1.3$ & $25.1 \pm 2.1^{*}$ & $28.2 \pm 1.4$ & $25.5 \pm 1.9$ & $29.3 \pm 2.3$ & $22.4 \pm 0.3 * *$ \\
\hline Latency () & $268.3 \pm 26.7$ & $373.7 \pm 40.0 * *$ & $255.5 \pm 12.4$ & $286.9 \pm 24.5$ & $250.5 \pm 31.4$ & $270.6 \pm 15.8$ \\
\hline \multicolumn{7}{|l|}{ CLE } \\
\hline Total time (min) & $10.8 \pm 1.0$ & $1.1 \pm 0.2^{\# \# \#}$ & $11.8 \pm 2.5$ & $11.5 \pm 2.7$ & $10.1 \pm 1.0$ & $22.5 \pm 1.2^{* *}$ \\
\hline Episode duration (s) & $118.4 \pm 8.2$ & $102.7 \pm 13.2$ & $148.9 \pm 10.7$ & $122.2 \pm 3.6$ & $119.5 \pm 6.2$ & $129.4 \pm 6.5$ \\
\hline Episode no. & $5.5 \pm 0.4$ & $0.6 \pm 0.1^{\# \# \#}$ & $5.0 \pm 1.3$ & $5.7 \pm 1.3$ & $5.0 \pm 0.4$ & $10.3 \pm 0.3 * *$ \\
\hline \multicolumn{7}{|l|}{ 4-h dark onset } \\
\hline \multicolumn{7}{|l|}{ Wakefulness } \\
\hline Total time (min) & $144.6 \pm 4.7$ & $97.1 \pm 6.7 * * *$ & $145.6 \pm 5.0$ & $121.8 \pm 13.0$ & $147.8 \pm 6.5$ & $143.5 \pm 3.5$ \\
\hline Episode duration (s) & $303.2 \pm 13.8$ & $198.4 \pm 7.5^{* *}$ & $290.3 \pm 10.5$ & $230.3 \pm 26.3$ & $305.2 \pm 19.1$ & $342.3 \pm 68.0$ \\
\hline Episode no. & $29.1 \pm 1.2$ & $29.9 \pm 1.5$ & $30.5 \pm 1.3$ & $32.3 \pm 0.9$ & $29.6 \pm 1.8$ & $30.6 \pm 4.2$ \\
\hline \multicolumn{7}{|l|}{ NREM sleep } \\
\hline Total time $(\mathrm{min})$ & $75.0 \pm 5.4$ & $125.6 \pm 8.7 * *$ & $68.8 \pm 6.3$ & $98.4 \pm 12.7$ & $68.2 \pm 6.5$ & $71.5 \pm 3.5$ \\
\hline Episode duration (s) & $169.1 \pm 17.1$ & $254.1 \pm 30.3^{*}$ & $144.1 \pm 12.9$ & $191.8 \pm 21.3$ & $152.4 \pm 19.7$ & $179.3 \pm 21.9$ \\
\hline Episode no. & $27.1 \pm 1.2$ & $31.6 \pm 1.7$ & $28.7 \pm 1.2$ & $31.3 \pm 0.9$ & $27.6 \pm 1.9$ & $25.8 \pm 3.9$ \\
\hline \multicolumn{7}{|l|}{ REM sleep } \\
\hline Total time $(\mathrm{min})$ & $15.1 \pm 1.6$ & $16.7 \pm 3.2$ & $17.2 \pm 0.6$ & $13.9 \pm 2.2$ & $19.1 \pm 0.8$ & $13.0 \pm 2.0$ \\
\hline Episode duration (s) & $97.0 \pm 6.0$ & $108.6 \pm 8.3$ & $97.3 \pm 4.0$ & $92.5 \pm 8.9$ & $105.8 \pm 6.1$ & $120.5 \pm 9.9$ \\
\hline Episode no. & $9.5 \pm 0.9$ & $9.0 \pm 1.5$ & $10.8 \pm 0.5$ & $9.1 \pm 0.9 *$ & $11.1 \pm 0.7$ & $6.6 \pm 0.7^{*}$ \\
\hline Latency (s) & $211.3 \pm 30.2$ & $383.9 \pm 69.0$ * & $181.8 \pm 24.7$ & $208.9 \pm 34.8$ & $185.4 \pm 22.9$ & $284.2 \pm 33.5$ \\
\hline \multicolumn{7}{|l|}{ CLE } \\
\hline Total time $(\mathrm{min})$ & $5.2 \pm 0.4$ & $0.5 \pm 0.2 * * *$ & $8.3 \pm 1.8$ & $5.7 \pm 1.1$ & $4.9 \pm 1.1$ & $11.3 \pm 0.9 * *$ \\
\hline Episode duration (s) & $114.1 \pm 8.3$ & $69.4 \pm 22.1 *$ & $159.6 \pm 14.0$ & $119.1 \pm 13.8$ & $105.5 \pm 15.0$ & $122.2 \pm 12.2$ \\
\hline Episode no. & $2.8 \pm 0.3$ & $0.3 \pm 0.1$ ** & $3.3 \pm 0.9$ & $3.0 \pm 0.6$ & $2.7 \pm 0.3$ & $5.7 \pm 0.4$ *** \\
\hline
\end{tabular}

EEG/EMG during the 12-h or 4-h period without chocolate feeding after CNO administration at the dark onset was analyzed. Values are mean \pm SEM, ${ }^{*} P<0.05, * * P<0.01,{ }^{* * *} P<0.001$, two-tailed Student's paired $t$ test; ${ }^{\# \# \#} P<0.001$, Welch's $t$ test. 
Table S5. Vigilance state parameters recorded from $0 \times 1 r^{-1-} 0 \times 2 r^{-1-}$ mice receiving the bilateral restoration of orexin receptors in the amygdala

\begin{tabular}{|c|c|c|c|}
\hline \multirow[b]{2}{*}{ Parameter } & \multicolumn{3}{|c|}{ Amygdala OX1R and OX2R $(n=3)$} \\
\hline & -Chocolate (4 h) & -Chocolate (12 h) & +Chocolate $(4 \mathrm{~h})$ \\
\hline \multicolumn{4}{|l|}{ Wakefulness } \\
\hline Total time (min) & $118.3 \pm 9.0$ & $347.8 \pm 7.0$ & $170.7 \pm 5.4$ \\
\hline Episode duration (s) & $327.3 \pm 43.1$ & $277.6 \pm 15.9$ & $397.7 \pm 39.9$ \\
\hline Episode no. & $21.3 \pm 1.2$ & $74.7 \pm 3.5$ & $25.7 \pm 3.5$ \\
\hline \multicolumn{4}{|l|}{ NREM sleep } \\
\hline Total time (min) & $102.9 \pm 7.9$ & $321.7 \pm 6.1$ & $45.5 \pm 7.3$ \\
\hline Episode duration (s) & $259.0 \pm 7.8$ & $249.8 \pm 13.9$ & $144.0 \pm 29.5$ \\
\hline Episode no. & $22.7 \pm 1.7$ & $76.9 \pm 3.6$ & $18.3 \pm 2.0$ \\
\hline \multicolumn{4}{|l|}{ REM sleep } \\
\hline Total time (min) & $17.7 \pm 2.4$ & $50.5 \pm 1.6$ & $8.3 \pm 3.0$ \\
\hline Episode duration (s) & $102.3 \pm 2.4$ & $98.2 \pm 3.9$ & $88.2 \pm 17.6$ \\
\hline Episode no. & $10.3 \pm 1.2$ & $30.9 \pm 2.1$ & $5.0 \pm 1.2$ \\
\hline Latency (s) & $327.7 \pm 27.4$ & $317.5 \pm 25.6$ & $161.0 \pm 53.4$ \\
\hline \multicolumn{4}{|l|}{ CLE } \\
\hline Total time (min) & $1.1 \pm 1.1$ & $4.7 \pm 2.1$ & $14.7 \pm 5.6$ \\
\hline Episode duration (s) & $68.0 \pm 68.0$ & $103.4 \pm 11.2$ & $150.4 \pm 33.4$ \\
\hline Episode no. & $0.3 \pm 0.3$ & $2.6 \pm 1.0$ & $7.3 \pm 3.3$ \\
\hline
\end{tabular}

Table S6. Vigilance state parameters recorded from $O \times 1 r^{-I-} O \times 2 r^{-1-}$ mice receiving the restoration of OX2R in DRN serotonin neurons and vSWO $\mathrm{S}_{5-\mathrm{HT} 1 \mathrm{~B}}$ stimulation at DRN projections in the amygdala

\begin{tabular}{lcc} 
& \multicolumn{2}{c}{ Amygdala vSWO $(n=4)$} \\
\cline { 2 - 3 } Parameter & -Light $(4 \mathrm{~h})$ & + Light $(4 \mathrm{~h})$ \\
\hline Wakefulness & & \\
$\quad$ Total time (min) & $3185.1 \pm 5.6$. & $164.7 \pm 5.8^{* *}$ \\
$\quad$ Episode duration (s) & $30.4 \pm 1.6$ & $231.7 \pm 16.0^{* *}$ \\
$\quad$ Episode no. & & $38.4 \pm 3.1^{*}$ \\
NREM sleep & $42.2 \pm 4.9$ & $60.2 \pm 5.1^{* * *}$ \\
$\quad$ Total time (min) & $79.0 \pm 6.8$ & $108.3 \pm 14.0^{*}$ \\
Episode duration (s) & $31.3 \pm 1.6$ & $34.9 \pm 2.8$ \\
$\quad$ Episode no. & $11.9 \pm 0.7$ & $9.2 \pm 1.6$ \\
REM sleep & $95.4 \pm 11.9$ & $77.2 \pm 5.5$ \\
$\quad$ Total time (min) & $8.0 \pm 1.0$ & $6.7 \pm 1.0$ \\
Episode duration (s) & $117.0 \pm 6.2$ & $133.7 \pm 21.0$ \\
$\quad$ Episode no. & & \\
Latency (s) & $0.7 \pm 0.4$ & $6.0 \pm 0.7^{* *}$ \\
CLE & $41.1 \pm 26.6$ & $4.7 \pm 0.5^{* * *}$ \\
$\quad$ Total time (min) & $0.3 \pm 0.2$ & \\
$\quad$ Episode duration (s) & & \\
Episode no. & &
\end{tabular}

EEG/EMG during the 4-h chocolate feeding was analyzed. Values are mean $\pm \mathrm{SEM}$, $* P<0.05,{ }^{*} P<0.01, * * * P<0.001$, two-tailed Student's paired $t$ test. 